\title{
A selective Nav1.1 activator with potential for treatment of Dravet syndrome epilepsy
}

\author{
Chun Yuen Chow ${ }^{\mathrm{a}}$, Yanni K.-Y. Chin ${ }^{\mathrm{a}}$, Linlin Ma ${ }^{\mathrm{a}, \#}$, Eivind A.B. Undheim ${ }^{\mathrm{b}, \AA}$, \\ Volker Herzig ${ }^{\mathrm{a}, \mathrm{Il}}$ and Glenn F. King ${ }^{\mathrm{a}, *}$
}

${ }^{a}$ Institute for Molecular Bioscience, The University of Queensland, Brisbane QLD 4072, Australia

${ }^{\mathrm{b}}$ Centre for Advanced Imaging, The University of Queensland, Brisbane QLD 4072, Australia

Current addresses:

\#Griffith Institute for Drug Discovery, Griffith University, Nathan, QLD 4111, Australia

Centre for Biodiversity Dynamics, Department of Biology, Norwegian University of Science and Technology, 7491, Trondheim, Norway

${ }^{\S}$ Centre for Ecological and Evolutionary Synthesis, Department of Bioscience, The University of Oslo, 0316, Oslo, Norway

IISchool of Science \& Engineering, University of the Sunshine Coast, Sippy Downs, QLD 4556, Australia.

*Address for correspondence:

Prof. Glenn F. King, Institute for Molecular Bioscience, The University of Queensland, 306 Carmody Road, St. Lucia, QLD 4072, Australia; E-mail: glenn.king@imb.uq.edu.au; Phone: +617 3346-2025.

Category that best describes this work: Neuropharmacology

Keywords: Nav1.1, voltage-gated sodium channel, venom peptide, Dravet syndrome, epilepsy 


\begin{abstract}
Dravet syndrome (DS) is a catastrophic epileptic encephalopathy characterised by childhood-onset polymorphic seizures, multiple neuropsychiatric comorbidities, and increased risk of sudden death. Heterozygous loss-of-function mutations in one allele of SCN1A, the gene encoding the voltagegated sodium channel 1.1 (Nav1.1), lead to DS. Nav1.1 is primarily found in the axon initial segment of fast-spiking GABAergic inhibitory interneurons in the brain, and the principle mechanism proposed to underlie seizure genesis in DS is loss of inhibitory input due to dysfunctional firing of GABAergic interneurons. We hypothesised that DS symptoms could be ameliorated by a drug that activates the reduced population of functional Nav1.1 channels in DS interneurons. We recently identified two homologous disulfide-rich spider-venom peptides (Hm1a and Hm1b) that selectively potentiate Nav1.1, and showed that selective activation of Nav1.1 by Hm1a restores the function of inhibitory interneurons in a mouse model of DS. Here we produced recombinant $\mathrm{Hm} 1 \mathrm{~b}(\mathrm{rHm} 1 \mathrm{~b})$ using an E. coli periplasmic expression system, and examined its selectivity against a panel of human Nav subtypes using whole-cell patch-clamp recordings. $\mathrm{rHm} 1 \mathrm{~b}$ is a potent and highly selective agonist of Nav1.1 and Nav1.3 ( $\mathrm{EC}_{50} \sim 12 \mathrm{nM}$ for both). $\mathrm{rHm} 1 \mathrm{~b}$ is a gating modifier that shifts the voltage dependence of channel activation and inactivation to hyperpolarised and depolarised potentials respectively, presumably by interacting with the channel's voltage-sensor domains. Like Hm1a, the structure of rHm1b determined by using NMR revealed a classical inhibitor cystine knot (ICK) motif. However, we show that rHm1b is an order of magnitude more stable than Hm1a in human cerebrospinal fluid. Overall, our data suggest that $\mathrm{rHm} 1 \mathrm{~b}$ is an exciting lead for a precision therapeutic targeted against DS.
\end{abstract}




\section{Introduction}

Voltage-gated sodium ( $\mathrm{Nav}$ ) channels play a fundamental role in normal neurological function, especially in the initiation and propagation of action potentials. They underpin diverse fundamental processes, including electrical signalling in the nervous system, skeletal and cardiac muscle contraction, and hormone secretion [1-3]. To date, nine Nav channel $\alpha$-subunits (denoted Nav1.11.9) have been cloned from mammals that share greater than $50 \%$ identity in their transmembrane (TM) and extracellular domains [4]. These channels are composed of four homologous but nonidentical domains (DI-DIV), with each domain containing six TM segments (S1-S6) [5, 6]. The S1-S4 segments constitute a voltage-sensing domain (VSD). A positively charged voltage sensor (located in the S4 segment) is driven outward in response to membrane depolarisation. All four VSDs are involved in channel activation, whereas the DIV VSD governs fast inactivation [7].

$\mathrm{Nav}$ channels have become major therapeutic targets for treatment of a range of nervous system disorders. The main subtypes expressed in the developing central nervous system (CNS) are Nav1.1, Nav1.2, Nav1.3, and Nav1.6. De novo loss-of-function mutations in one allele of SCN1A, the gene encoding the Nav1.1, cause Dravet syndrome (DS) [8,9]. DS is a catastrophic epileptic encephalopathy associated with childhood-onset polymorphic seizures, often precipitated by a small increase in body temperature [10]. Patients with DS subsequently develop multiple neuropsychiatric comorbidities, including cognitive deficits, psychomotor regression, autistic traits, and increased risk of premature death [11-13]. One of the defining characteristics of DS is that it is resistant to most antiepileptic drugs (AEDs). Because of the small population of affected patients and variable phenotypic severity, clinical trials of novel therapies are challenging. Thus far, two drugs have been approved by the U.S. Food and Drug Administration for treatment of DS - cannabidiol and stiripentol-however, both of these are limited to symptomatic management of epileptic seizures $[14,15]$. There is therefore an urgent need to develop effective therapeutics to target the underlying pathogenesis with minimal side effects.

Studies of SCN1A knockout and knock-in mouse models demonstrated that DS is caused by reduced excitability of GABAergic inhibitory interneurons without a corresponding change in the activity of excitatory neurons [16-18]. In the brain, Nav1.1 is located at the axon initial segments (AISs) of fast-spiking GABAergic inhibitory interneurons, which are responsible for sending inhibitory signals to excitatory cells $[19,20]$. In mammalian neurons, the AIS bridges dendritic and axonal compartments, and converts the dendritic inputs into all-or-none APs [21]. Consequently, the abnormal levels of functional Nav1.1 prevent different classes of GABAergic inhibitory interneurons from firing properly, which is thought to underlie seizure genesis in DS [22, 23]. It has 
been proposed that upregulating the reduced population of functional Nav1.1 channels in DS might rescue the firing of inhibitory interneurons as it targets the underlying molecular deficit [24].

Spider venoms have proven to be an invaluable pool of ion channel modulators, including many toxins with high potency and selectivity for specific Nav channel subtypes [25-27]. While conducting a screen to identify spider-venom peptides that activate mouse sensory neurons, Osteen et al. (2016) discovered two toxins from venom of the tarantula Heteroscodra maculata that selectively activate the human Nav1.1 channel expressed in Xenopus oocytes [28]. These two toxins were named as $\delta$-theraphotoxin Hm1a and $\delta$-theraphotoxin-Hm1b (henceforth Hm1a and Hm1b, respectively). These two toxins are new members of the extended family of inhibitor cystine knot (ICK) peptides with $\mathrm{C}_{1}-\mathrm{C}_{4}, \mathrm{C}_{2}-\mathrm{C}_{5}$ and $\mathrm{C}_{3}-\mathrm{C}_{6}$ disulfide architecture [29]. It was subsequently shown that $\mathrm{Hm} 1 \mathrm{a}$ restores the function of inhibitory interneurons from DS mice in vitro, and eliminates seizures in a mouse model of DS [30]. However, Hm1a is unstable in cerebrospinal fluid (CSF), indicating that bolus administration might only provide short-term relief from seizures. Here, we examined the therapeutic potential of the homologous peptide Hm1b. Patch-clamp electrophysiology revealed that $\mathrm{Hm} 1 \mathrm{~b}$ is a highly potent and selective modulator of $\mathrm{Nav} 1.1\left(\mathrm{EC}_{50}\right.$ $12 \mathrm{nM}$ ). Despite the high degree of sequence similarity between $\mathrm{Hm} 1 \mathrm{a}$ and $\mathrm{Hm} 1 \mathrm{~b}$, the latter is much more stable in biological fluids. The three-dimensional structure of Hm1b was also determined using NMR spectroscopy. This study provides a useful starting point for delivering therapies that will positively impact DS patients, and will guide discoveries of novel therapeutic leads for other SCN1A-related epileptic syndromes.

\section{Methods and materials}

\section{Venom fractionation}

Lyophilised venom from $H$. maculata was obtained by electrical stimulation [31], and fractionated using reversed-phase high performance liquid chromatography (RP-HPLC) on a Prominence HPLC system (Shimadzu Scientific Instruments, Rydalmere, NSW, Australia). Venom was loaded onto a $\mathrm{C}_{18}$ column (Kinetex [Phenomenex, CA, USA]; $250 \times 4.6 \mathrm{~mm}, 5 \mu \mathrm{m}$ ) with the following gradient: $5 \%$ solvent $\mathrm{B}(0.043 \%$ trifluoroacetic acid [TFA] in $90 \%$ acetonitrile $[\mathrm{MeCN}])$ in solvent $\mathrm{A}(0.05 \%$ TFA in ultrapure water) for $5 \mathrm{~min}$, followed by $5-15 \%$ solvent B over $5 \mathrm{~min}, 15-40 \%$ solvent B over $30 \mathrm{~min}$, then $40-80 \%$ solvent B for $5 \mathrm{~min}$ (flow rate $1 \mathrm{~mL} / \mathrm{min}$ ). A secondary purification step was performed using a Pursuit Diphenyl column (Agilent; $150 \times 3 \mathrm{~mm}, 3 \mu \mathrm{m}$ ) with a linear gradient of $20-40 \%$ solvent $B$ in solvent A over $40 \mathrm{~min}$ (flow rate $0.6 \mathrm{~mL} / \mathrm{min}$ ). Identities of native (n) $\mathrm{Hm} 1 \mathrm{a}$ and $\mathrm{nHm} 1 \mathrm{~b}$ were confirmed by matrix-assisted laser desorption/ionisation time-of-flight mass spectrometry (MALDI-TOF MS) using a 5800 Proteomics Bioanalyser (Applied Biosystems, 
CA, USA) operated in positive reflector mode with a laser power of 3,800-4,000 V. RP-HPLC fractions were mixed 1:1 v/v with $\alpha$-cyano-4-hydroxy-cinnamic acid (CHCA; Sigma-Aldrich, St. Louis, MO, USA) matrix (7.5 mg/mL in $50 \% \mathrm{MeCN}, 1 \% \mathrm{TFA})$ and spotted onto a MALDI target plate. All reported masses are for monoisotopic $[\mathrm{M}+1 \mathrm{H}]^{1+}$ ions.

\section{Recombinant production of $\mathrm{Hm} 1 \mathrm{~b}$}

Recombinant $\mathrm{Hm} 1 \mathrm{~b}$ (henceforth $\mathrm{rHm} 1 \mathrm{~b}$ ) was produced using an E. coli periplasmic expression system as previously described [32, 33]. A synthetic gene encoding the peptide was subcloned into a pLIC-MBP (maltose binding protein) expression vector as a commercial service by GeneArt ${ }^{\mathrm{TM}}$ (Invitrogen, Regensburg, Germany). Cultures were grown in Lysogeny broth (LB) medium containing $100 \mu \mathrm{g} / \mathrm{mL}$ ampicillin at $37^{\circ} \mathrm{C}$ with shaking at $160 \mathrm{rpm}$. Toxin gene expression was induced with $250 \mu \mathrm{M}$ isopropyl $\beta$-D-1-thiogalactopyranoside (IPTG, Bioline, Eveleigh, NSW, Australia) at $\mathrm{OD}_{600}=0.8-1.2$, then cells were grown at $16^{\circ} \mathrm{C}$ overnight before collecting by centrifugation for $15 \mathrm{~min}$ at 6,000 rpm. The His6-MBP-toxin fusion protein was extracted from the bacterial periplasm by cell disruption at $28 \mathrm{kpsi}$, followed by a second round at $32 \mathrm{kpsi}$ (TS Series Cell Disrupter, Constant Systems Ltd, Northants, UK), then captured by passing the clear cell lysate (buffered in TN buffer: $20 \mathrm{mM}$ Tris-HCl, $200 \mathrm{mM} \mathrm{NaCl}, \mathrm{pH}$ 8.0) over Ni-NTA Superflow resin (Qiagen, Chadstone, Australia). The resin was washed with TN buffer containing $15 \mathrm{mM}$ imidazole ( $\mathrm{pH}$ 8.0) to remove weakly bound proteins then the toxin fusion protein was eluted using TN buffer containing $400 \mathrm{mM}$ imidazole ( $\mathrm{pH}$ 8.0). The fusion protein was concentrated to $5 \mathrm{~mL}$, then cleaved overnight at room temperature with tobacco etch virus (TEV) protease in the presence of $5 \mathrm{~mL}$ TN buffer ( $\mathrm{pH}$ 8.0) containing reduced and oxidised glutathione (0.6 mM GSH and $0.4 \mathrm{mM}$ GSSG, respectively; Sigma-Aldrich). The liberated recombinant peptide was then isolated using RP-HPLC on a semi-preparative $\mathrm{C}_{4}$ column (Jupiter [Phenomenex]; $250 \times 10 \mathrm{~mm}$, $10 \mu \mathrm{m}$ ) with a linear gradient of $40-50 \%$ solvent B in solvent A over 20 min (flow rate 2 $\mathrm{mL} / \mathrm{min})$. Subsequent purification to obtain a single isoform was performed using an analytical $\mathrm{C}_{18}$ column (Aeris [Phenomenex]; $250 \times 4.6 \mathrm{~mm}, 3.6 \mu \mathrm{m}$ ) with a linear gradient of 30-40\% solvent B in solvent A over $20 \mathrm{~min}$ (flow rate $0.75 \mathrm{~mL} / \mathrm{min}$ ). Fractions were collected manually by monitoring eluent absorption at $214 \mathrm{~nm}$ and $280 \mathrm{~nm}$. All solvents used were HPLC grade.

\section{NMR structure determination of rHm1b}

Uniformly ${ }^{15} \mathrm{~N}$-labelled $\mathrm{rHm} 1 \mathrm{~b}$ was produced using almost the same protocol as described above for production of unlabelled peptide. Cells were grown in $2 \mathrm{~L}$ of LB media, then cells were harvested by centrifugation at $6,000 \mathrm{rpm}$ for $15 \mathrm{~min}$ at $4{ }^{\circ} \mathrm{C}$. Media was poured off and the cell pellet was gently resuspended in $500 \mathrm{~mL}$ of modified M9 minimal media [34] comprising $22 \mathrm{mM} \mathrm{KH}_{2} \mathrm{PO}_{4}$, 
$90 \mathrm{mM} \mathrm{Na}_{2} \mathrm{HPO}_{4}$ and $17 \mathrm{mM} \mathrm{NaCl}$ supplemented with $1.6 \mathrm{mM} \mathrm{MgSO}_{4}, 80 \mathrm{nM} \mathrm{CaCl}, 18 \mathrm{mM}$

${ }^{15} \mathrm{NH}_{4} \mathrm{Cl}$ (as the sole nitrogen source), $22 \mathrm{mM}$ D-glucose, $1 \mathrm{mg}$ of thiamine, BME vitamin solution (Sigma-Aldrich), and $100 \mu \mathrm{g} / \mathrm{mL}$ ampicillin. The cultures were further incubated at $37^{\circ} \mathrm{C}$ for $2 \mathrm{~h}$ with shaking at $160 \mathrm{rpm}$. Protein expression was induced with $250 \mu \mathrm{M}$ IPTG and the culture was left at $16^{\circ} \mathrm{C}$ overnight with shaking. Purified, lyophilised peptide was then reconstituted at a concentration of $325 \mu \mathrm{M}$ in NMR buffer (20 mM sodium phosphate, $\mathrm{pH} 6.0 ; 95 \% \mathrm{H}_{2} \mathrm{O}, 5 \% \mathrm{D}_{2} \mathrm{O}$ ), and $300 \mu \mathrm{L}$ of sample was aliquoted into a susceptibility-matched $5 \mathrm{~mm}$ outer diameter microtube (Shigemi Inc., PA, USA) for NMR experiments.

NMR spectra were acquired at $25^{\circ} \mathrm{C}$ on a Bruker AVANCE III HD $600 \mathrm{MHz}$ NMR spectrometer equipped with a cryogenically-cooled triple resonance probe (Bruker BioSpin, Bremen, Germany). Sequence-specific resonance assignments were obtained using a combination of $2 \mathrm{D}{ }^{1} \mathrm{H}-{ }^{15} \mathrm{~N}$ HSQC, 2D ${ }^{1} \mathrm{H}-{ }^{13} \mathrm{C}$ HSQC, 2D ${ }^{1} \mathrm{H}-{ }^{1} \mathrm{H}$ TOCSY, 2D ${ }^{1} \mathrm{H}-{ }^{1} \mathrm{H}$ NOESY and 3D ${ }^{1} \mathrm{H}-{ }^{15} \mathrm{~N}$ TOCSY-HSQC spectra. The mixing times used for the TOCSY and NOESY spectra were 80 and $250 \mathrm{~ms}$, respectively. Spectra were processed using TopSpin (V3.5.b.91 pl 7; Bruker BioSpin) and analysed using CcpNmr Analysis (V2.4.1) [35]. Chemical shift assignments have been deposited in BioMagResBank (BMRB) with accession code 30695. The disulfide-bond framework was determined from dipolar connectivities in 2D spectra [36]. NOESY spectra were assigned and 200 structures calculated automatically (i.e., without manual intervention) using the CYANA software package [37]. The 30 conformers with the lowest CYANA target function values in the final round of structure calculations were subsequently analysed using MolProbity [38], and the 20 conformers with highest stereochemical quality were selected to represent the solution structure of $\mathrm{rHm} 1 \mathrm{~b}$. The structures statistics are shown in Table 1 and atomic coordinates for the final ensemble of 20 structures have been deposited in the PDB with accession code 6V6T.

\section{Biological stability of nHm1a and rHm1b}

Stability assays were performed using the protocol previously described for nHm1a [30]. Briefly, the stability of $\mathrm{rHm} 1 \mathrm{~b}$ was analysed using male AB human serum (Sigma-Aldrich) and human CSF collected from healthy volunteers (acquired from The Centre for Motor Neuron Disease Research, Macquarie University, NSW, Australia). Both serum and CSF were prewarmed to $37^{\circ} \mathrm{C}$ for $15 \mathrm{~min}$. Approximately $1.4 \mu \mathrm{g}$ of $\mathrm{rHm} 1 \mathrm{~b}$ was added to human serum and a pooled CSF sample (final concentration $0.5 \mu \mathrm{M}$ ), then the samples were incubated at $37^{\circ} \mathrm{C}$ for periods up to $72 \mathrm{~h}$. Triplicate samples were collected at $0,1,2,3,4,8,12,24,48$, and $72 \mathrm{~h}$. The reaction mixture was precipitated at the desired time by adding $5 \mu \mathrm{l}$ of 5\% TFA and centrifuging the sample at 23,000 $\mathrm{g}$ for $10 \mathrm{~min}$ at $4^{\circ} \mathrm{C}$. The integrity of $\mathrm{rHm} 1 \mathrm{~b}$ in the supernatant was then analysed with LC-MS using a $\mathrm{C}_{18}$ column 
(Kinetex [Phenomenex]; $150 \mathrm{~mm} \times 2.1 \mathrm{~mm}, 2.6 \mu \mathrm{m}$ ) with a linear gradient of 2-40\% mobile phase B $(0.1 \%$ formic acid $[\mathrm{FA}]$ in $90 \% \mathrm{MeCN})$ in mobile phase $\mathrm{A}(0.1 \% \mathrm{FA})$ at a flow rate of $0.2 \mathrm{~mL} / \mathrm{min}$ over $14 \mathrm{~min}$ coupled to a TripleTOF 5600 mass spectrometer (AB SCIEX, Framingham, MA, USA) with a cycle time of 0.2751 s. A similar approach was used to examine the stability of nHm1a in human serum. Peptide areas were measured at triple-, quadruple- and quintuple-charge states, and analysed using PeakView and MultiQuant (AB SCIEX, Framingham, MA, USA). Peptide abundance at each time point was then calculated and normalised to the initial time point, and the data fitted using a two-phase exponential decay function. Human atrial natriuretic peptide (hANP, $1 \mu \mathrm{M}$ ) from GenScript (Piscataway, NJ, USA) and ziconotide (also known as Prialt, $1 \mu \mathrm{M}$ ) from Alomone Labs (Jerusalem, Israel) served as controls.

\section{Cell culture}

All cell culture reagents used in this study were from Gibco (Life Technologies Corporation, Carlsbad, CA, USA) unless stated otherwise. HEK293 cells stably expressing human Nav1.1-1.7 along with the $\beta 1$ auxiliary subunit (SB Drug Discovery, Glasgow, UK) were cultured in T75 flasks at $37^{\circ} \mathrm{C}$ in a humidified $5 \% \mathrm{CO}_{2}$ incubator in Dulbecco's modified Eagle's medium supplemented with $10 \% \mathrm{v} / \mathrm{v}$ heat inactivated fetal bovine serum (Assay Matrix, Melbourne, VIC, Australia), 1× GlutaMAX, 1× MEM Non-essential Amino Acid Solution, and variable concentrations of blasticidin, geneticin and zeocin according to the supplier's protocols. Cells were resuspended in fresh culture medium and transferred to T75 flask (for maintenance) and T175 flask (for assays) in ratio of 1:5 and 1:3, respectively. Cells having undergone less than 20 passages were used for experiments.

\section{Patch-clamp electrophysiology}

Patch-clamp experiments were conducted using a QPatch-16X automated whole-cell patch-clamp electrophysiology system (Sophion Bioscience, Ballerup, Denmark) at room temperature $\left(\sim 24^{\circ} \mathrm{C}\right)$. The extracellular solution comprised (in $\mathrm{mM}$ ): $2 \mathrm{CaCl}_{2}, 1 \mathrm{MgCl}_{2}, 10 \mathrm{HEPES}, 4 \mathrm{KCl}, 145 \mathrm{NaCl}, 10$ sucrose, pH 7.4 with $\mathrm{NaOH}$, and 305 mOsm. The intracellular solution comprised (in $\mathrm{mM}$ ): 140 CsF, 1 EGTA, $5 \mathrm{CsOH}, 10$ HEPES, $10 \mathrm{NaCl}$, pH 7.4 with $\mathrm{CsOH}$, and $320 \mathrm{mOsm}$. The current elicited were digitised at $25 \mathrm{kHz}$ and filtered at $4 \mathrm{kHz}$. Series resistance did not exceed $5 \mathrm{M} \Omega$ and was at least $80 \%$ compensated. Leak-subtraction protocol was applied with non-leak-subtracted currents acquired in parallel. Cells with currents less than $1 \mathrm{nA}$ or greater than $5 \mathrm{nA}$ were excluded. Cells were maintained at a holding potential of $-80 \mathrm{mV}$, followed by a pre-pulse of $-120 \mathrm{mV}$ for $200 \mathrm{~ms}$ and then a 50-ms step depolarisation to $0 \mathrm{mV}$. To obtain concentration-response curves, 
cells were incubated for at least 3 min with increasing concentrations of peptides in extracellular solution containing $0.1 \% \mathrm{w} / \mathrm{v}$ bovine serum albumin. Data were fitted with the Hill equation:

$$
\frac{I}{I_{\max }}=\frac{[\mathrm{A}]^{n_{\mathrm{H}}}}{\mathrm{EC}_{50}+[\mathrm{A}]^{n_{\mathrm{H}}}}
$$

where $I$ is the sustained current at the end of a 20 -ms depolarising pulse normalised to the peak currents $\left(I_{\text {sustained }} / I_{\text {peak }}\right),[\mathrm{A}]$ is the peptide concentration, $\mathrm{EC}_{50}$ is the concentration of agonist producing $50 \%$ of the maximal current, and $n_{\mathrm{H}}$ is the slope factor. All values are given as mean \pm s.e.m. for the number of independent measurements indicated $(n)$.

\section{Mode of action of $\mathbf{r H m} 1 \mathrm{~b}$}

To examine the voltage dependence of activation of Nav1.1 channels, cells were held from a holding potential of $-120 \mathrm{mV}$ following a $100-\mathrm{ms}$ depolarising pulse from $-120 \mathrm{mV}$ to $+30 \mathrm{mV}$ in $5-\mathrm{mV}$ increments. The conductance-voltage $(G-V)$ relationship was determined by measuring the peak sodium conductance, which was determined using:

$$
G=\frac{I_{\text {peak }}}{V-V_{\text {rev }}}
$$

where $I_{\text {peak }}$ is the peak current during the step voltage and $V_{\text {rev }}$ is the calculated reversal potential for sodium ion, which is $67.55 \mathrm{mV}$. The calculated conductance value at each voltage step was normalised to maximum conductance $\left(G_{\max }\right)$ plotted against the test voltage, and fitted with the Boltzmann equation:

$$
\frac{G}{G_{\max }}=\frac{1}{1+\mathrm{e}^{\frac{V_{\text {half act }}-V}{k_{\text {act }}}}}
$$

where $V_{\text {half act }}$ is the half-maximal activation potential and $k_{\text {act }}$ is the slope factor of activation.

Steady-state fast inactivation of Nav1.1 was measured using a two-step protocol: starting from a holding potential of $-120 \mathrm{mV}$, pre-pulses were given to $+30 \mathrm{mV}$ for $500 \mathrm{~ms}$ in $5-\mathrm{mV}$ increments, and then the level of inactivation was tested by subjecting to $0 \mathrm{mV}$ for $20 \mathrm{~ms}$. The peak current amplitude during the test pulse was normalised to the maximum current amplitude $\left(I_{\max }\right)$ plotted against the pre-pulse potential, and fitted with the Boltzmann function: 


$$
\frac{I}{I_{\max }}=\frac{1}{1+\mathrm{e}^{\frac{V-V_{\text {half inact }}}{k_{\text {inact }}}}}
$$

where $V_{\text {half inact }}$ is the half-maximal inactivation potential and $k_{\text {inact }}$ is the slope factor of inactivation.

Recovery from fast inactivation was studied by pre-pulsing the cells to $0 \mathrm{mV}$ for $30 \mathrm{~ms}$ (conditioning pulse) to inactivate all of the currents, and then recovery potential to $-120 \mathrm{mV}$ for an interval of variable durations $(\Delta t)$ was applied before depolarising to $0 \mathrm{mV}$ for $30 \mathrm{~ms}$ (test pulse). The ratio of the peak amplitude evoked by the test pulse to that evoked by the conditioning pulse $\left(I_{\text {tess }} / I_{\text {conditioning }}\right)$ was normalised to the maximum amplitude $\left(I_{\text {test_max }} / I_{\text {conditioning_max }}\right)$ plotted against the duration time between the two pulses, and fitted with the double-exponential function:

$$
\frac{\left(I_{\text {test }} / I_{\text {conditioning }}\right)}{\left(I_{\text {test_max }} / I_{\text {conditioning_max }}\right)}=A_{\text {fast }} \times \mathrm{e}^{\frac{-t}{\tau_{\text {fast }}}}+A_{\text {slow }} \times \mathrm{e}^{\frac{-t}{\tau_{\text {slow }}}}+I_{0}
$$

where $t$ is time, $I_{0}$ is the non-inactivating component, and $A_{\text {fast }}$ and $A_{\text {slow }}$ are the components for the fast and slow recovery time constants ( $\tau_{\text {fast }}$ and $\tau_{\text {slow }}$, respectively).

\section{Results}

\section{Isolation of nHm1a and nHm1b}

RP-HPLC fractionation using a $\mathrm{C}_{18}$ column was used to purify nHm1a and $\mathrm{nHm} 1 \mathrm{~b}$ from the venom of $H$. maculata (Fig. 1a); the resulting chromatogram revealed that $H$. maculata venom is highly complex (Fig. 1b). A subsequent purification step using a diphenyl column led to the isolation of nHm1a and nHm1; the observed monoisotopic masses (for the $[\mathrm{M}+1 \mathrm{H}]^{1+}$ ion) of 3,995.51 Da for nHm1a and 3,892.23 Da for nHm1b (Fig. 1c,d inset) were consistent with the predicted monoisotopic masses of 3,995.61 Da and 3,892.65 Da, respectively. To date, twelve families of spider-venom peptides that modulate Nav channels (so called NaSpTx) have been described [27] based on amino acid sequence similarity and disulfide-bond connectivity. Sequence alignments revealed that $\mathrm{nHm} 1 \mathrm{a}$ and $\mathrm{nHm} 1 \mathrm{~b}$ belong to NaSpTx family 2, which is the largest family of spider toxins with predicted Nav channel inhibitory function. The sequence identity between nHmla and $\mathrm{nHm} 1 \mathrm{~b}$ is $79 \%$ (Fig. 1d,e), reflecting their similar activities as Nav1.1 agonists. As revealed by a combination of Edman degradation and carboxypeptide $\mathrm{Y}$ digestion [28], nHm1b has 34 amino acid residues plus an amidated carboxy terminus.

\section{Production of rHm1b}


Due to the limited amount of crude venom, we first produced correctly folded rHm1b (Fig. 2a) using a cost- and time-effective method for pharmacological characterisation as well as isotopic labeling for NMR-based structure determination. This was achieved by utilising an IPTG-inducible construct (Fig. 2b) that allows production of a His6-MBP-Hm1b fusion protein in the periplasm of E. coli. Note that, in addition to lacking the C-terminal amidation present in $\mathrm{nHm} 1 \mathrm{~b}, \mathrm{rHm} 1 \mathrm{~b}$ contains a non-native N-terminal Ser residue that is a vestige of the TEV protease recognition site used to cleave $\mathrm{rHm} 1 \mathrm{~b}$ from the His $6_{-} \mathrm{MBP}$ fusion tag (Fig. 2b). The fusion protein was robustly overexpressed after IPTG induction as indicated by an intense band at approximately $45 \mathrm{kDa}$ (Fig. 2c, lane 2). After isolation of the soluble cell fraction, the fusion protein was purified using nickel affinity chromatography (Fig. 2c, lanes 5-8) then cleaved with TEV protease to liberate rHm1b (compare lanes 8 and 9 in Fig. 2c) Subsequent RP-HPLC purification using a $\mathrm{C}_{4}$ column (Fig. 2d) followed by a $\mathrm{C}_{18}$ column (Fig. 2e) yielded a major peak corresponding to the native disulfide-bond isomer). MALDI-TOF MS analysis of $\mathrm{rHm} 1 \mathrm{~b}$ revealed a monoisotopic mass of 3,980.47 Da (Fig. 2f), in accordance with the predicted theoretical mass of 3,980.67 Da for the recombinant peptide. However, the yield of $\mathrm{rHm} 1 \mathrm{~b}$ was low $(50 \mu \mathrm{g} / \mathrm{L}$ bacterial culture $)$ compared to other peptide toxins produced in-house using the same method [32]. We investigated various conditions for optimising $\mathrm{rHm} 1 \mathrm{~b}$ production, including induction temperature, IPTG concentration, and the composition of the redox buffer used for TEV cleavage. Unfortunately, none of these variations yielded a significant improvement in the yield of $\mathrm{rHm} 1 \mathrm{~b}$.

\section{Nav channel selectivity profile of $\mathbf{r H m} 1 \mathrm{~b}$}

Next we assessed the selectivity of rHm1b against human Nav1.1-1.7 channels stably expressed in HEK293 cells using automated whole-cell patch-clamp electrophysiology (Fig. 3a). rHm1b $(300 \mathrm{nM})$ robustly slowed the time course of fast inactivation for Nav1.1 and Nav1.3, as determined by the fraction of sodium current remaining $5 \mathrm{~ms}$ after the peak, relative to the peak current $\left(I_{5 \mathrm{~ms}} / I_{\text {peak }}\right)(\mathrm{P}<0.0005$ and $\mathrm{P}<0.005$, respectively; Fig. $3 \mathrm{~b})$. This provides an indication of the proportion of channels that are not inactivated after $5 \mathrm{~ms}$. rHm1b had no effect on other Nav channel subtypes aside from minor inhibition of fast inactivation of Nav1.5 and Nav1.7 (both P < 0.05; Fig. 3b). However, for these two channel subtypes, the resulting currents for a depolarising step to $0 \mathrm{mV}$ were completely decayed to zero within $\sim 10 \mathrm{~ms}$ (i.e., rHm1b did not induce a persistent current; Fig. 3c).

In addition to slowing channel inactivation, $\mathrm{rHm} 1 \mathrm{~b}$ inhibited transition of Nav1.1 and Nav1.3 to a completely inactivated state, leading to sustained currents at the end of the test pulse (Fig. 3a). The sustained Nav1.1 and Nav1.3 currents evoked by rHm1b were concentration-dependent (Fig. 3d). 
Concentration-response analysis showed that $\mathrm{rHm} 1 \mathrm{~b}$ is an equipotent agonist of Nav1.1 and Nav1.3 with half-maximal effective concentration $\left(\mathrm{EC}_{50}\right)$ values of $11.6 \pm 3.5 \mathrm{nM}$ and $11.8 \pm 1.8 \mathrm{nM}$, respectively (Fig. 3e). Although $\mathrm{rHm} 1 \mathrm{~b}$ has similar potency on Nav1.1 and Nav1.3, the sustained current for Nav1.1 was significantly higher than that of Nav1.3 in the presence of $300 \mathrm{nM} \mathrm{rHm} 1 \mathrm{~b}$ (P < 0.0005; Fig. 3f). Even at this high concentration (i.e., 30 times the $\mathrm{EC}_{50}$ at Nav1.1 and Nav1.3), rHm1b did not induce persistent currents in any other Nav channel subtypes.

At a saturating concentration of $300 \mathrm{nM}, \mathrm{rHm} 1 \mathrm{~b}$ increased the peak current amplitudes of Nav1.1 and Nav1.3 by $32.3 \pm 2.7 \%$ and $34.6 \pm 1.9 \%$, respectively (Fig. $3 \mathrm{~h}$ ). In addition, it caused inhibition of Nav1.5 and Nav1.7 peak currents (27.0 $\pm 0.7 \%$ and $27.4 \pm 1.1 \%$, respectively). Similar inhibition of peak currents was also seen for Nav1.2, Nav1.4 and Nav1.6 $(17.1 \pm 1.2 \%, 16.0 \pm 1.2 \%$ and 17.3 $\pm 1.1 \%$, respectively). Taken together, our data indicate that $\mathrm{rHm} 1 \mathrm{~b}$ is a highly potent and selective modulator of Nav1.1 and Nav1.3; on these channels the peptide slows fast inactivation, increases peak current, and prevents a fraction of the sodium current from becoming inactivated.

\section{Nav selectivity profile of $\mathbf{n H m 1 b}$}

In addition to $\mathrm{rHm} 1 \mathrm{~b}$, we also quantified the effect of $\mathrm{nHm} 1 \mathrm{~b}$ on all available human Nav channel subtypes expressed in HEK293 cells. $\mathrm{nHm} 1 \mathrm{~b}$ was found to have a similar selectivity profile to $\mathrm{rHm} 1 \mathrm{~b}$ (Fig. 4a). At a saturating concentration of $300 \mathrm{nM}, \mathrm{nHm} 1 \mathrm{~b}$ significantly slowed the inactivation of human $\mathrm{Nav} 1.1, \mathrm{Nav} 1.3$, Nav1.5, and Nav1.7 (Fig. 4b), as determined by the $I_{5 \mathrm{~ms}} / I_{\text {peak }}$ ratio. As for rHm1b, Nav1.5 and Nav1.7 currents decayed to zero within about 15 ms (Fig. 4c). Concentration-response analysis revealed that $\mathrm{nHm} 1 \mathrm{~b}(1 \mu \mathrm{M})$ increased the peak current amplitudes of Nav1.1 and Nav1.3 by $17.7 \pm 4.5 \%$ and $22.2 \pm 3.2 \%$, respectively (Fig. 4d). Surprisingly, in striking contrast with $\mathrm{rHm} 1 \mathrm{~b}, \mathrm{nHm} 1 \mathrm{~b}$ delayed Nav1.2 inactivation (Fig. 4b,c), and inhibited currents by up to $50 \%$, with a half-maximal inhibitory concentration ( $\mathrm{IC}_{50}$ ) of $21.6 \pm 0.8 \mathrm{nM}$ (Fig. 4d). This difference in pharmacology must be due to the additional N-terminal Ser residue in $\mathrm{rHm} 1 \mathrm{~b}$ and/or the lack of C-terminal amidation. $\mathrm{nHm} 1 \mathrm{~b}$ increased sustained currents for Nav1.1 and Nav1.3 in a concentration-dependent manner, with $\mathrm{EC}_{50}$ values of $12.1 \pm 1.8 \mathrm{nM}$ and $10.7 \pm 2.8 \mathrm{nM}$, respectively (Fig. 4e). In a similar fashion, the sustained current for Nav1.1 was two-fold greater than that of Nav1.3 in the presence of $300 \mathrm{nM} \mathrm{nHm1b}(\mathrm{P}<0.005$; Fig. 4f).

\section{Mode of action of rHm1b}

To better understand the mechanisms underlying the ability of $\mathrm{rHm} 1 \mathrm{~b}$ to increase both peak and sustained currents in human $\mathrm{Nav} 1.1$, we examined the effect of $\mathrm{rHm} 1 \mathrm{~b}$ on the gating properties of Nav1.1. rHm1b induced a hyperpolarising shift in the voltage dependence of activation (Fig. 5a), 
causing a significant shift $(-9.2 \mathrm{mV})$ in the half-maximal activation potential $(\mathrm{P}<0.0005$; Fig. 5c) without a change in the slope of the conductance-voltage relationship (Fig. 5d). This suggests that $\mathrm{rHm} 1 \mathrm{~b}$ would lower the threshold for action potential firing, resulting in an increase in the fraction of Nav1.1 channels that open in response to a given depolarisation. rHm1b also induced a depolarising shift in the voltage dependence of steady-state inactivation (Fig. 5b). The inactivation curve was shifted to less negative potentials $(\mathrm{P}<0.05$; Fig. $5 \mathrm{c})$, indicating that, at any given potential, the probability of Nav1.1 channel inactivation is decreased, consistent with the sustained currents evoked by rHm1b. rHm1b also caused a significant reduction in the slope of the inactivation curve ( $\mathrm{P}<0.05$; Fig. 5d).

Due to the shifts it induced in the voltage dependence of activation and inactivation, $\mathrm{rHm} 1 \mathrm{~b}$ introduced a large overlap between the activation and steady-state inactivation curves. This would result in a broad window current within the physiologically relevant voltage range (Fig. 5e). At about $-35 \mathrm{mV}$, the peak window current probability predicts that a small percentage of Nav1.1 channels will be spontaneously activated but not inactivate completely (with $\sim 30 \%$ maximal conductance). Taken together, the data indicate that $\mathrm{rHm} 1 \mathrm{~b}$ increases $\mathrm{Na}_{\mathrm{v}} 1.1$ channel availability due to destabilisation of the fast-inactivated state. We next investigated whether $\mathrm{rHm} 1 \mathrm{~b}$ alters the inactivation decay time constants. When $\mathrm{Na}_{V}$ channels enter the inactivated state in a timedependent transition, the onset of fast inactivation can be described by fast and slow inactivation time constants obtained by fitting the decay phase of the current trace to a double-exponential function. A significant increase in fast- and slow-inactivating time constants was observed for Nav1.1, Nav1.3, Nav1.5, and Nav1.7 (Fig. 5f,g,h), consistent with previous findings that $\mathrm{rHm} 1 \mathrm{~b}$ delayed the time course of fast inactivation.

Finally, we examined whether $\mathrm{rHm} 1 \mathrm{~b}$ affects recovery of Nav1.1 currents from fast inactivation. $\mathrm{Nav}$ channels experience a latency period in which they recover from the inactivated state to the closed state. This transition, which is more rapid at hyperpolarising potentials, occurs before the channels are capable of opening in response to subsequent depolarisations. The sustained current was observed at all recovery times with $\mathrm{rHm} 1 \mathrm{~b}$ treatment (Fig. 6a). Most Nav1.1 channels (>80\%) recovered from fast inactivation within $\sim 5 \mathrm{~ms}$ under both conditions (Fig. 6b). At $300 \mathrm{nM}, \mathrm{rHm} 1 \mathrm{~b}$ accelerates recovery from inactivation as indicated by the significantly smaller time constants for both the fast and slow components $(\mathrm{P}<0.0005$ and $\mathrm{P}<0.005$, respectively; Fig. 6c). These experiments, along with the results described above, indicate that at any given potential within the physiological range, $\mathrm{rHm} 1 \mathrm{~b}$ promotes Nav1.1 activation and inhibits fast inactivation. 


\section{Biological stability of nHm1a and rHm1b}

To evaluate the biological stability of $\mathrm{rHm} 1 \mathrm{~b}$, the peptide was incubated in human CSF and serum at $37^{\circ} \mathrm{C}$ for $72 \mathrm{~h}$. Despite the poor in vivo stability of nHm1a in CSF as previously described [30], we also elucidated its stability in human serum. For comparison, we included the analgesic drug ziconotide, a highly stable ICK peptide [39], as positive control, and hANP, which is known to be unstable in vivo [40], as a negative control. As expected, hANP was rapidly degraded in human CSF and human serum, with less than $\sim 10 \%$ remaining after $4 \mathrm{~h}$ (Fig. 7a,b). Remarkably, despite the high degree of sequence similarity between $\mathrm{nHm} 1 \mathrm{a}$ and $\mathrm{rHm} 1 \mathrm{~b}$, we found that $\mathrm{rHm} 1 \mathrm{~b}$ was much more stable than nHmla and even more stable than ziconotide in both human CSF and human serum. The half-life of $\mathrm{rHm} 1 \mathrm{~b}$ in human serum was greater than $40 \mathrm{~h}$, compared to $\sim 17 \mathrm{~h}$ for ziconotide and $\sim 10 \mathrm{~h}$ for $\mathrm{nHm} 1 \mathrm{a}$ (Fig. $7 \mathrm{~b}$ ). The difference was more dramatic in human CSF, where rHm1b had a half-life $>70 \mathrm{~h}$ (Fig. 7a) compared to $\sim 1.7 \mathrm{~h}$ for nHm1a [30].

\section{Three-dimensional structure of $\mathbf{r H m} 1 \mathrm{~b}$}

We produced uniformly ${ }^{15} \mathrm{~N}$-labelled $\mathrm{rHm} 1 \mathrm{~b}$ for NMR studies by expression in the periplasm of E. coli. The peak dispersion in the fully assigned ${ }^{15} \mathrm{~N}-\mathrm{HSQC}$ NMR spectrum of $\mathrm{rHm} 1 \mathrm{~b}$ indicates that the peptide adopts a globular fold as opposed to being structurally disordered. We observed the expected number of 37 amide protons, including 34 backbone amide peaks (i.e., one from each residue, except the $\mathrm{N}$-terminal Ser), plus three peaks from the side-chain amide groups of the single Trp residue and two Arg residues. Automatic assignment of the NOESY spectrum using CYANA yielded 499 structurally relevant interproton distance restraints. These were combined with nine disulfide-bond restraints ( 3 per disulfide) plus 53 dihedral-angle restraints for structure calculations. The final ensemble of $20 \mathrm{rHm} 1 \mathrm{~b}$ structures has very high precision with a root mean square deviation (RMSD) of $0.06 \AA$ over the backbone atoms of the ordered regions of the peptide (residues 3-33) (Table 1). The ensemble of structures also has high stereochemical quality, with a mean MolProbity score of 1.51 (among the top 95 percentile). The disulfide-bond connectivity was unambiguously determined from preliminary structure calculations performed without disulfidebond restraints; the intercystine NOEs in the NOESY spectrum [36] are consistent with the pattern Cys3-Cys17, Cys10-Cys22 and Cys16-Cys29 (highlighted in yellow; Fig. 8a,b). The dominant secondary structural feature is a $\beta$ hairpin (residues 19-32) comprising His19-Gly21 ( $\beta 1$ ) and Ala30-Asp32 ( $\beta 2)$ (highlighted in orange in Fig. 8a,b). Overall, the tertiary structure of rHm1b conforms to the ICK topology, in which the Cys16-Cys29 disulfide bond pierces a loop formed by the Cys3-Cys17 and Cys10-Cys22 disulfide bonds and the intervening section of the polypeptide backbone. 
The molecular surface of $\mathrm{rHm} 1 \mathrm{~b}$ has a dispersed distribution of charged residues (Fig. 9b), but there is a distinct cluster of hydrophobic residues (except Phe35) on one 'face' of the peptide (Fig. 9c). Sequence alignments revealed that nHm1a and $\mathrm{rHm} 1 \mathrm{~b}$ differ by only seven residues (Fig. 9a, indicated by green shading). Most of these residues (except Leu26) are located on the opposite face to the hydrophobic patch (Fig. 9d). We previously suggested that the hydrophobic residues of nHm1a (i.e., Tyr5, Leu6, Phe7, Tyr28, Trp31, and Phe35) likely mediate its interaction with Nav1.1 channels [30]. Given the pharmacological similarity between Hm1a and Hm1b [28], we conclude that the conserved hydrophobic patch in rHm1b (i.e., Tyr5, Leu6, Phe7, Leu26, Tyr28, and Trp31; Fig. 9a, indicated by red shading) is likely to mediate its interaction with Nav1.1. Note that the Leu residue at position 20 is completely buried in nHm1a and $\mathrm{rHm} 1 \mathrm{~b}$, and therefore it is unlikely to be involved in the interaction with Nav1.1.

$\mathrm{Hm} 1 \mathrm{a} / \mathrm{Hm} 1 \mathrm{~b}$ have a high level of sequence similarity with other tarantula-venom peptides, and presumably share the same disulfide connectivities as well as tertiary structure characterised by a cystine knot. The most similar toxin is $\kappa$-TRTX-Scg1a (SGTx1), a 34 amino acid-long peptide from Scodra griseipes venom that binds to the VSD of voltage-gated potassium channel $\mathrm{K}_{\mathrm{V}} 2.1$ [41]. Both nHm1a and rHm1b contain two $\beta$ strands comprised of Ser21-Arg23 and Tyr28-Ala30 (for nHm1a), and His19-Gly21 and Ala30-Asp32 (for rHm1b) (Fig. 10a); these residues stabilise the $\beta$ hairpins through a network of hydrogen-bond interactions (Fig. 10c, left and middle panels). Similarly, SGTx1 contains two $\beta$ strands comprised of Leu20-Arg23 and Tyr28-Trp31 (Fig. 10c, right panel [41]). Comparison of the tertiary structure of these three toxins revealed several differences in the $\beta$ hairpins. First, the antiparallel $\beta$ strands of nHm1a and SGTx 1 are located further away from the C-terminal region (indicated by cyan and magenta arrows, respectively; Fig. 10b), whereas that of $\mathrm{rHm} 1 \mathrm{~b}$ is located nearer the C-terminus (indicated by orange arrows; Fig. 10b). Second, nHm1a and SGTx1 contain a four-residue $\beta$-hairpin loop (Fig. 10c, left and right panels), whereas $\mathrm{rHm} 1 \mathrm{~b}$ has a longer eight-residue loop (Fig. 10c, middle panel). Overall, nHm1a has a long, flexible C-terminal chain (Trp31-Ser36) compared to nHm1b and SGTx1 (Fig. 10a).

\section{Discussion}

\section{Hm1b inhibits the gating movement of the domain IV voltage sensor}

In the present study, we demonstrated that both $\mathrm{nHm} 1 \mathrm{~b}$ and $\mathrm{rHm} 1 \mathrm{~b}$ enhance Nav1.1 currents and delay fast inactivation of the channel, leading to a sustained current. Similar effects were observed at Nav1.3, but with less sustained currents. Our findings differ from those previously reported for nHm1b, which was shown to have no effect on Nav1.2, Nav1.3, Nav1.5, and Nav1.7 channels [28]. These previous data were obtained using oocytes expressing human $\mathrm{Nav}$ channel $\alpha$-subunits in the 
absence of auxiliary $\beta$-subunits, whereas we used mammalian cells expressing human Nav channel $\alpha$-subunits along with $\beta 1$ subunit. These data indicate that the $\beta 1$ subunit and/or the heterologous expression system have a significant impact on the pharmacology of nHm1b.

Previous studies showed that spider-venom peptides that target the DIV VSD delay fast inactivation, whereas peptides that target the DI-DIII VSDs influence channel opening [42]. Experiments in which the S3b-S4 paddle motif of each VSD of Nav1.1 was transplanted into homotetrameric rat $\mathrm{K}_{\mathrm{V}} 2.1$ channel revealed that $\mathrm{Hm} 1 \mathrm{a}$ (and by extension Hm1b) interacts with the DIV VSD [28]. However, this segment of the DIV VSD is not sufficient to impart peptide selectivity because the DIV S3-S4 loop is identical or highly conserved among human Nav channel subtypes. Subsequent mutational analysis revealed that the DIV S1-S2 loop is a primary determinant of $\mathrm{Hm} 1 \mathrm{a} / \mathrm{Hm} 1 \mathrm{~b}$ subtype selectivity [28]. $\mathrm{rHm} 1 \mathrm{~b}$ causes a hyperpolarising shift in the voltage dependence of Nav1.1 activation, whereas $\mathrm{nHm} 1 \mathrm{a}$ does not [30]. This indicates that the channels activate more readily in the presence of $\mathrm{rHm} 1 \mathrm{~b}$, suggesting a possible additional interaction with the DII VSD [43]. Together, the differences in their sequences might indicate which residues in $\mathrm{Hm} 1 \mathrm{~b}$ are responsible for the stronger gating modulation.

The electrophysiological data and the sequence conservation between Hm1a and Hm1b revealed that the hydrophobic face of rHm1b interacts with the DIV VSD of Nav1.1 and Nav1.3 and traps the voltage-sensing S4 helix in a partially activated state, distinguishing it from certain $\alpha$-scorpion toxins that trap the DIV-S4 in the deactivated conformation (i.e., in the inward-facing position) [44]. Thus, the channels would maintain a persistent open state but not sufficient to trigger inactivation. When the DIV VSD is fully activated (i.e., S4 is in the fully extracellular-facing 'outward' or 'up' position), it removes the steric hindrance that prevents closure of the inactivation gate. Numerous insect-selective toxins isolated from spiders and scorpions are known to interfere the activation and inactivation processes. Examples include $\beta / \delta$-agatoxins isolated from Agelena orientalis and Ts1 isolated from Tityus serrulatus, both of which induce sustained currents in a bellshaped concentration- and voltage-dependent manner [45, 46]. The mechanism underlying the ability of these toxins to delay inactivation at negative potentials could be explained by a voltagesensor trapping mechanism. As for $\beta / \delta$-agatoxins and Ts 1 , we speculate that $\mathrm{rHm} 1 \mathrm{~b}$ binds to the DIV VSD with high affinity.. Overall, the functional outcome of this mode of action is that $\mathrm{rHm} 1 \mathrm{~b}$ makes Nav1.1 and Nav1.3 difficult to enter the inactivated state and thereby evokes a noninactivating sustained current.

Sequence alignments reveal that $\mathrm{Hm} 1 \mathrm{a}$ and $\mathrm{Hm} 1 \mathrm{~b}$ share highest sequence similarity with other 
tarantula peptides, including $\kappa$-TRTX-Gr1a (HaTx1), and $\tau / \kappa-T R T X-P c 1 a(V a T x 1)$. These toxins belong to NaSpTx family 2. Their activity at human Nav1.1 remains to be determined, but they have been reported to modulate the gating of ion channels other than Nav channels. HaTx1, isolated from the venom of the Chilean rose tarantula Grammostola rosea, is a gating modifier that inhibits $\mathrm{K}_{\mathrm{V}} 2.1$ [47]. Site-directed mutagenesis revealed that HaTx1 targets the extracellular S3-S4 linkers and stabilises the VSD in a resting conformation [48, 49]. The other closely related toxin, VaTx1 from the venom of the Trinidad chevron tarantula Psalmopoeus cambridgei, activates transient receptor potential vanilloid 1 (TRPV1) and induces inflammatory pain [50]. Bohlen et al. (2010) determined that VaTx1 exerts its influence through the channel's outer pore region and traps TRPV1 in an open state [51]. However, H. maculata venom has no effect on TRPV1 [50], and therefore $\mathrm{Hm} 1 \mathrm{a}$ and $\mathrm{Hm} 1 \mathrm{~b}$ are unlikely to have significant activity on this channel.

\section{nHm1b also inhibits Nav1.2 currents}

Peptide post-translational modifications (PTMs) are often used by venomous creatures to further diversify their toxic arsenals. In contrast to cone snails, which make extensive use of PTMs [52], spiders make relatively few modifications to their venom peptides. Aside from disulfide bridges, the most common PTM in spider-venom peptides is C-terminal amidation, which is generated by $\mathrm{C}$ terminal trimming of a Gly residue and simultaneous formation of a carboxamide $\left(-\mathrm{CONH}_{2}\right)$ at the preceding amino acid residue. In addition to providing protection from exopeptidase cleavage, Cterminal amidation can alter biological activity. For example, the native amidated version of the 35residue spider-venom peptide $\mu$-theraphotoxin-Hs2a (also known as HwTx-IV), a member of NaSpTx family 1, inhibits human Nav1.7 with 50-fold higher potency than a recombinant version with a C-terminal carboxylate group [53].

Our results demonstrated an important distinction between $\mathrm{nHm} 1 \mathrm{~b}$ and $\mathrm{rHm} 1 \mathrm{~b}$; nHm1b, but not $\mathrm{rHm} 1 \mathrm{~b}$, inhibits $\mathrm{Nav} 1.2$ and has minor effects on inactivation at moderate nanomolar concentrations. Drugs that activate Nav1.2, the primary Nav subtype in excitatory neurons, would be contraindicated in most epilepsies. Indeed, missense mutations in Nav1.2 that enhance channel activation or inhibit inactivation are the underlying cause of several neonatal-infantile onset epilepsies [54, 55]. However, since Nav1.2 is primarily localised to the axon initial segments of excitatory neurons, Nav1.2 inhibition in combination with a Nav1.1 agonist might be beneficial in DS as both pharmacologies would help to reduce brain hyperexcitability.

\section{Biological stability of rHm1b}

The tertiary structures of Hm1a and Hm1b are similar; they have the same disulfide connectivity, 
both form an ICK motif, and they have the same secondary structural feature, namely an antiparallel $\beta$ hairpin. Nevertheless, despite their similarities, they have dramatically different stability in human serum and CSF. This is likely due to intrinsic differences in their amino acid sequence (they differ at eight positions), and the greater flexibility of the C-terminal region of $\mathrm{nHm} 1 \mathrm{a}$, which is one-residue longer than $\mathrm{rHm} 1 \mathrm{~b}$ (Fig. 2.10a). A recent study with spider-derived ICK peptides revealed that the longer C-terminal chain of SGTx1 (Ala30-Phe35; Fig. 2.10a) makes it susceptible to proteolysis, with $\sim 50 \%$ remaining intact after $24 \mathrm{~h}$ in human serum [56]. There are numerous approaches that could be considered to improve the stability of nHm1a, including replacement of disulfide bonds with diselenide bonds, substitution of L-amino acids with D-amino acids, and N- to $\mathrm{C}$ terminal cyclisation. The latter has previously been reported to increase the protease resistance of disulfide-rich peptides while maintaining biological activity [57, 58]. As such, it would be of interest to examine whether backbone cyclisation could improve the biological stability of nHm1a without affecting potency, selectivity, and efficacy.

The in vivo half-life of Hmla in CSF indicates that repeated doses or continuous infusion would be required to maintain a high enough concentration of nHm1a in the brain to be therapeutically effective. In the present study, we demonstrated that $\mathrm{rHm} 1 \mathrm{~b}$ has exceptional CSF stability compared to $\mathrm{nHm} 1 \mathrm{a}$. Thus, we predict that $\mathrm{rHm} 1 \mathrm{~b}$ will have a significantly longer therapeutic time window than Hm1a (i.e., a bolus dose would produce a much longer seizure-free period).

\section{Development of Hm1a and Hm1b as antiepileptic drugs}

Several studies have demonstrated a progressive postnatal decline in Nav1.3 expression and a concomitant increase in Nav1.1 expression during development of normal human brain [59, 60]. This suggests that the loss of Nav1.3 during brain development and the lack of a compensatory increase in functional Nav1.1 channels are coupled to seizure onset in DS patients, and it explains why seizures typically do not present until 5-8 months after. Thus, early diagnosis of DS by molecular genetics would help to prevent inappropriate treatments (e.g., Nav channel blockers) and drive timely provision of personalised therapy. Since $\mathrm{rHm} 1 \mathrm{~b}$ enhances Nav1.1 and to a lesser extent Nav1.3, it could be used at a lower dose prior to seizure onset to avoid overstimulation of inhibitory interneurons, then the dose could be increased gradually after seizure onset to counteract the loss of Nav1.1 function. Extensive animal studies are required to test this hypothesis and optimize the therapeutic strategy.

As well as being expressed in the brain, Nav1.1 is also expressed in peripheral A $\delta$ sensory neurons, where it is involved in the transduction of mechanical pain [28]. As such, Nav1.1-selective 
activators may elevate pain sensitivity. However, DS patients suffer from Nav1.1 haploinsufficiency (i.e., they have only $~ 50 \%$ of normal Nav1.1 channel), and there is anecdotal evidence that they have high mechanical pain thresholds; hence Nav1.1 activators may not be pronociceptive in DS patients depending on the systemic concentration of the drug. One advantage of a centrally-administered antiepileptic peptide such as Hm1a is that they would be unlikely to reach peripheral neurons, consistent with DS mice showing no adverse effects [30]. Thus, delivery of Hm1a directly to the brain, rather than systemic delivery, might be a suitable approach for treatment of DS epilepsy.

From a clinical perspective, two major concerns that need to be considered for therapeutic application of $\mathrm{Hm} 1 \mathrm{a} / \mathrm{Hm} 1 \mathrm{~b}$ (and by extension other Nav1.1-selective agonists) are the route of administration and optimal dosage. Drug administration via the CSF theoretically allows direct access to the brain; however, the actual drug penetration from the CSF into the brain tissue is often limited, due to the slow diffusion and efflux of drug to the blood compartment via bulk flow of CSF [61]. This also raises an unresolved question of what concentration is needed to be therapeutically effective for preventing seizures. Although the imbalance between excitation and inhibition contributes to hyperexcitability in DS patients, on-target overdosing with a Nav1.1 agonist might lead to overstimulation of inhibitory neuron activity resulting in sedation or, in the extreme case, coma or death. As mentioned above, DS treatment would require to be delivered on a routine basis, rather than a one-time injection. This remains challenging with the long-term use of intracerebroventricular (ICV) devices, including complications with infections and catheter maintenance [62]. Thus, while ICV delivery of peptidic Nav1.1 agonists is an option for treatment of DS, it is not an easy route of administration compared to currently available oral medications and intravenous administration. Nevertheless, the extremely poor quality of life of the most severely affected DS patients, and the lack of effective medications, might warrant such an approach.

\section{Acknowledgments}

Funding for this work was provided by Citizen's United for Research in Epilepsy (Pediatrics Award 353711 to G.F.K.), The University of Queensland (International Postgraduate Scholarship to C.Y.C.), the Australian Research Council (Discovery Grant DE160101142 to E.A.B.U.), the Australian National Health \& Medical Research Council (Principal Research Fellowship APP1136889 to G.F.K.), and the Norwegian Research Council (FRIPRO-YRT Fellowship no. 287462 to E.A.B.U.). Mass spectrometry analyses were conducted at the Institute for Molecular Bioscience Proteomics Facility, The University of Queensland. We thank the members of the Deutsche Arachnologische Gesellschaft, particularly Ingo Wendt, Jörg Broghammer, Arnd 
Schlosser, Bastian Rast, Michelle Lüscher, Claudia and Frank Schneider and Hans-Wener Auer for providing $H$. maculata for milking. 


\section{References}

[1] Catterall WA, Structure and function of voltage-gated sodium channels at atomic resolution, Exp Physiol 99 (2014) 35-51.

[2] George AL, Jr., Inherited disorders of voltage-gated sodium channels, J Clin Invest 115 (2005) 19901999.

[3] Stojilkovic SS, Tabak J, Bertram R, Ion channels and signaling in the pituitary gland, Endocr Rev 31 (2010) 845-915.

[4] Catterall WA, Goldin AL, Waxman SG, International Union of pharmacology. XLVII. Nomenclature and structure-function relationships of voltage-gated sodium channels, Pharmacol Rev 57 (2005) 397409.

[5] Noda M, Shimizu S, Tanabe T, Takai T, Kayano T, Ikeda T, et al., Primary structure of Electrophorus electricus sodium channel deduced from cDNA sequence, Nature 312 (1984) 121-127.

[6] Sato C, Ueno Y, Asai K, Takahashi K, Sato M, Engel A, et al., The voltage-sensitive sodium channel is a bell-shaped molecule with several cavities, Nature 409 (2001) 1047-1051.

[7] Armstrong CM, Na channel inactivation from open and closed states, Proc Natl Acad Sci USA 103 (2006) 17991-17996.

[8] Claes L, Del-Favero J, Ceulemans B, Lagae L, Van Broeckhoven C, De Jonghe P, De novo mutations in the sodium-channel gene SCN1A cause severe myoclonic epilepsy of infancy, Am J Hum Genet 68 (2001) 1327-1332.

[9] Depienne C, Trouillard O, Saint-Martin C, Gourfinkel-An I, Bouteiller D, Carpentier W, et al., Spectrum of SCN1A gene mutations associated with Dravet syndrome: analysis of 333 patients, J Med Genet 46 (2009) 183-191.

[10] Dravet C, The core Dravet syndrome phenotype, Epilepsia 52 Suppl 2 (2011) 3-9.

[11] Guzzetta F, Cognitive and behavioral characteristics of children with Dravet syndrome: an overview, Epilepsia 52 Suppl 2 (2011) 35-38.

[12] Kearney J, Sudden unexpected death in Dravet syndrome, Epilepsy Curr 13 (2013) 264-265.

[13] Nabbout R, Chemaly N, Chipaux M, Barcia G, Bouis C, Dubouch C, et al., Encephalopathy in children with Dravet syndrome is not a pure consequence of epilepsy, Orphanet J Rare Dis 8 (2013) 176.

[14] Devinsky O, Cross JH, Wright S, Trial of cannabidiol for drug-resistant seizures in the Dravet syndrome, N Engl J Med 377 (2017) 699-700.

[15] Perez J, Chiron C, Musial C, Rey E, Blehaut H, d'Athis P, et al., Stiripentol: efficacy and tolerability in children with epilepsy, Epilepsia 40 (1999) 1618-1626.

[16] Cheah CS, Yu FH, Westenbroek RE, Kalume FK, Oakley JC, Potter GB, et al., Specific deletion of $\mathrm{Na}_{\mathrm{v}} 1.1$ sodium channels in inhibitory interneurons causes seizures and premature death in a mouse model of Dravet syndrome, Proc Natl Acad Sci USA 109 (2012) 14646-14651.

[17] Tai C, Abe Y, Westenbroek RE, Scheuer T, Catterall WA, Impaired excitability of somatostatin- and parvalbumin-expressing cortical interneurons in a mouse model of Dravet syndrome, Proc Natl Acad Sci USA 111 (2014) 3139-3148. 
[18] Yu FH, Mantegazza M, Westenbroek RE, Robbins CA, Kalume F, Burton KA, et al., Reduced sodium current in GABAergic interneurons in a mouse model of severe myoclonic epilepsy in infancy, Nat Neurosci 9 (2006) 1142-1149.

[19] Duflocq A, Le Bras B, Bullier E, Couraud F, Davenne M, Nav1.1 is predominantly expressed in nodes of Ranvier and axon initial segments, Mol Cell Neurosci 39 (2008) 180-192.

[20] Wimmer VC, Reid CA, So EY, Berkovic SF, Petrou S, Axon initial segment dysfunction in epilepsy, J Physiol 588 (2010) 1829-1840.

[21] Hedstrom KL, Ogawa Y, Rasband MN, AnkyrinG is required for maintenance of the axon initial segment and neuronal polarity, J Cell Biol 183 (2008) 635-640.

[22] Kalume F, Yu FH, Westenbroek RE, Scheuer T, Catterall WA, Reduced sodium current in Purkinje neurons from $\mathrm{Na}_{\mathrm{v}} 1.1$ mutant mice: implications for ataxia in severe myoclonic epilepsy in infancy, $\mathrm{J}$ Neurosci 27 (2007) 11065-11074.

[23] Rubinstein M, Westenbroek RE, Yu FH, Jones CJ, Scheuer T, Catterall WA, Genetic background modulates impaired excitability of inhibitory neurons in a mouse model of Dravet syndrome, Neurobiol Dis 73 (2015) 106-117.

[24] Jensen HS, Grunnet M, Bastlund JF, Therapeutic potential of Nav1.1 activators, Trends Pharmacol Sci 35 (2014) 113-118.

[25] King GF, Escoubas P, Nicholson GM, Peptide toxins that selectively target insect $\mathrm{Na}_{\mathrm{v}}$ and $\mathrm{Ca}_{\mathrm{v}}$ channels, Channels 2 (2008) 100-116.

[26] King GF, Venoms as a platform for human drugs: translating toxins into therapeutics, Expert Opin Biol Ther 11 (2011) 1469-1484.

[27] Klint JK, Senff S, Rupasinghe DB, Er SY, Herzig V, Nicholson GM, et al., Spider-venom peptides that target voltage-gated sodium channels: pharmacological tools and potential therapeutic leads, Toxicon 60 (2012) 478-491.

[28] Osteen JD, Herzig V, Gilchrist J, Emrick JJ, Zhang C, Wang X, et al., Selective spider toxins reveal a role for Nav1.1 channel in mechanical pain, Nature 534 (2016) 494-499.

[29] Saez NJ, Senff S, Jensen JE, Er SY, Herzig V, Rash LD, et al., Spider-venom peptides as therapeutics, Toxins 2 (2010) 2851-2871.

[30] Richards KL, Milligan CJ, Richardson RJ, Jancovski N, Grunnet M, Jacobson LH, et al., Selective Nav1.1 activation rescues Dravet syndrome mice from seizures and premature death, Proc Natl Acad Sci USA 115 (2018) e8077-8085.

[31] Guo S, Herzig V, King GF, Dipteran toxicity assays for determining the oral insecticidal activity of venoms and toxins, Toxicon 150 (2018) 297-303.

[32] Klint JK, Senff S, Saez NJ, Seshadri R, Lau HY, Bende NS, et al., Production of recombinant disulfiderich venom peptides for structural and functional analysis via expression in the periplasm of $E$. coli, PLoS ONE 8 (2013) e63865.

[33] Saez NJ, Cristofori-Armstrong B, Anangi R, King GF, A strategy for production of correctly folded disulfide-rich peptides in the periplasm of E. coli, Methods Mol Biol 1586 (2017) 155-180. 
[34] Marley J, Lu M, Bracken C, A method for efficient isotopic labeling of recombinant proteins, J Biomol NMR 20 (2001) 71-75.

[35] Vranken WF, Boucher W, Stevens TJ, Fogh RH, Pajon A, Llinas M, et al., The CCPN data model for NMR spectroscopy: development of a software pipeline, Proteins 59 (2005) 687-696.

[36] Mobli M, King GF, NMR methods for determining disulfide-bond connectivities, Toxicon 56 (2010) 849-854.

[37] Guntert P, Automated NMR structure calculation with CYANA, Methods Mol Biol 278 (2004) 353378.

[38] Chen VB, Arendall WB, 3rd, Headd JJ, Keedy DA, Immormino RM, Kapral GJ, et al., MolProbity: allatom structure validation for macromolecular crystallography, Acta Crystallogr D Biol Crystallogr 66 (2010) 12-21.

[39] McGivern JG, Ziconotide: a review of its pharmacology and use in the treatment of pain, Neuropsychiatr Dis Treat 3 (2007) 69-85.

[40] Nelesen RA, Dimsdale JE, Ziegler MG, Plasma atrial natriuretic peptide is unstable under most storage conditions, Circulation 86 (1992) 463-466.

[41] Lee CW, Kim S, Roh SH, Endoh H, Kodera Y, Maeda T, et al., Solution structure and functional characterization of SGTx1, a modifier of KV2.1 channel gating, Biochemistry 43 (2004) 890-897.

[42] Bosmans F, Martin-Eauclaire MF, Swartz KJ, Deconstructing voltage sensor function and pharmacology in sodium channels, Nature 456 (2008) 202-208.

[43] Xiao Y, Blumenthal K, Cummins TR, Gating-pore currents demonstrate selective and specific modulation of individual sodium channel voltage-sensors by biological toxins, Mol Pharmacol 86 (2014) 159-167.

[44] Catterall WA, Cestele S, Yarov-Yarovoy V, Yu FH, Konoki K, Scheuer T, Voltage-gated ion channels and gating modifier toxins, Toxicon 49 (2007) 124-141.

[45] Billen B, Vassilevski A, Nikolsky A, Debaveye S, Tytgat J, Grishin E, Unique bell-shaped voltagedependent modulation of $\mathrm{Na}^{+}$channel gating by novel insect-selective toxins from the spider Agelena orientalis, J Biol Chem 285 (2010) 18545-18554.

[46] Peigneur S, Cologna CT, Cremonez CM, Mille BG, Pucca MB, Cuypers E, et al., A gamut of undiscovered electrophysiological effects produced by Tityus serrulatus toxin 1 on Nav-type isoforms, Neuropharmacology 95 (2015) 269-277.

[47] Swartz KJ, MacKinnon R, An inhibitor of the $K_{\mathrm{V}} 2.1$ potassium channel isolated from the venom of a Chilean tarantula, Neuron 15 (1995) 941-949.

[48] Lee HC, Wang JM, Swartz KJ, Interaction between extracellular Hanatoxin and the resting conformation of the voltage-sensor paddle in $K_{V}$ channels, Neuron 40 (2003) 527-536.

[49] Swartz KJ, MacKinnon R, Mapping the receptor site for hanatoxin, a gating modifier of voltagedependent $\mathrm{K}^{+}$channels, Neuron 18 (1997) 675-682.

[50] Siemens J, Zhou S, Piskorowski R, Nikai T, Lumpkin EA, Basbaum AI, et al., Spider toxins activate the capsaicin receptor to produce inflammatory pain, Nature 444 (2006) 208-212. 
[51] Bohlen CJ, Priel A, Zhou S, King D, Siemens J, Julius D, A bivalent tarantula toxin activates the capsaicin receptor, TRPV1, by targeting the outer pore domain, Cell 141 (2010) 834-845.

[52] Bergeron ZL, Chun JB, Baker MR, Sandall DW, Peigneur S, Yu PY, et al., A 'conovenomic' analysis of the milked venom from the mollusk-hunting cone snail Conus textile-the pharmacological importance of post-translational modifications, Peptides 49 (2013) 145-158.

[53] Sermadiras I, Revell J, Linley JE, Sandercock A, Ravn P, Recombinant expression and in vitro characterisation of active huwentoxin-IV, PLoS ONE 8 (2013) e83202.

[54] Lauxmann S, Boutry-Kryza N, Rivier C, Mueller S, Hedrich UB, Maljevic S, et al., An SCN2A mutation in a family with infantile seizures from Madagascar reveals an increased subthreshold $\mathrm{Na}^{+}$ current, Epilepsia 54 (2013) e117-121.

[55] Scalmani P, Rusconi R, Armatura E, Zara F, Avanzini G, Franceschetti S, et al., Effects in neocortical neurons of mutations of the Nav1.2 $\mathrm{Na}^{+}$channel causing benign familial neonatal-infantile seizures, $\mathrm{J}$ Neurosci 26 (2006) 10100-10109.

[56] Agwa AJ, Huang YH, Craik DJ, Henriques ST, Schroeder CI, Lengths of the C-terminus and interconnecting loops impact stability of spider-derived gating modifier toxins, Toxins 9 (2017) 248.

[57] Clark RJ, Akcan M, Kaas Q, Daly NL, Craik DJ, Cyclization of conotoxins to improve their biopharmaceutical properties, Toxicon 59 (2012) 446-455.

[58] Clark RJ, Fischer H, Dempster L, Daly NL, Rosengren KJ, Nevin ST, et al., Engineering stable peptide toxins by means of backbone cyclization: Stabilization of the $\alpha$-conotoxin MII, Proc Natl Acad Sci USA 102 (2005) 13767-13772.

[59] Cheah CS, Westenbroek RE, Roden WH, Kalume F, Oakley JC, Jansen LA, et al., Correlations in timing of sodium channel expression, epilepsy, and sudden death in Dravet syndrome, Channels 7 (2013) 468-472.

[60] Smith RS, Kenny CJ, Ganesh V, Jang A, Borges-Monroy R, Partlow JN, et al., Sodium channel SCN3A (Nav1.3) regulation of human cerebral cortical folding and oral motor development, Neuron 99 (2018) 905-913.

[61] Pardridge WM, CSF, blood-brain barrier, and brain drug delivery, Expert Opin Drug Deliv 13 (2016) 963-975.

[62] Cohen-Pfeffer JL, Gururangan S, Lester T, Lim DA, Shaywitz AJ, Westphal M, et al., Intracerebroventricular delivery as a safe, long-term route of drug administration, Pediatr Neurol 67 (2017) 23-35.

[63] Eisenberg D, Schwarz E, Komaromy M, Wall R, Analysis of membrane and surface protein sequences with the hydrophobic moment plot, J Mol Biol 179 (1984) 125-142. 


\section{Figures legends}

Fig. 1. Isolation of nHm1a and nHm1b. (a) Heteroscodra maculata, a tarantula native to West Africa (image courtesy of Bastian Rast). (b) RP-HPLC chromatogram showing fractionation of H. maculata venom. Peaks containing $\mathrm{nHm} 1 \mathrm{a}$ and $\mathrm{nHm} 1 \mathrm{~b}$ are shaded in red and blue, respectively. (c) $\mathrm{nHm} 1 \mathrm{a}$ and $\mathrm{nHm} 1 \mathrm{~b}$ were subsequently purified to homogeneity using a diphenyl column. MS analysis revealed that these venom peptides have monoisotopic masses of 3,995.51 Da (nHm1a, left panel) and 3,892.23 Da (nHm1b, right panel). (d) Alignment of nHm1a and nHm1b with closely related ICK peptides from Psalmopoeus cambridgei (VaTx1), Cyriopagopus schmidti (HwTx-V), Stromatopelma calceatum griseipes (SGTx1), Chilobrachys guangxiensis (JzTx-Xi), and Grammostola rosea (HaTxl). Cysteine residues characteristic of ICK peptides are shown in bold, and other highly conserved residues are highlighted in grey. The black bar indicates the hypervariable region. Asterisks denote C-terminal amidation. (e) Comparison of $\mathrm{nHm} 1 \mathrm{~b}$ with ICK peptides showing percentage amino acid identities and similarities.

Fig. 2. Recombinant production of $\mathbf{r H m 1 b}$. (a) A non-native N-terminal Ser residue was added to optimise TEV protease cleavage efficiency as the N-terminal residue of $n \mathrm{Hm} 1 \mathrm{~b}$ (Glu) is a nonpreferred residue for the P1' site of TEV protease. Cysteine residues are highlighted in bold. (b) Schematic representation of the pLIC vector used for periplasmic expression of $\mathrm{rHm} 1 \mathrm{~b}$. The coding region includes a ribosome-binding site (RBS), MalE signal sequence (MalEss), His ${ }_{6}$ affinity tag, MBP fusion tag, and a codon-optimised gene encoding Hm1b, with a TEV protease recognition site inserted between the MBP and toxin-coding regions. (c) SDS-PAGE gel demonstrating different steps in the purification of rHm1b. Lanes contain: M, molecular weight markers; 1, E. coli cell extract before IPTG induction; 2, E. coli cell extract after IPTG induction; 3, soluble cell extract; 4, insoluble cell extract after cell disruption; 5, flow-through from Ni-NTA column; 6, first wash from Ni-NTA resin with $15 \mathrm{mM}$ imidazole; 7, second wash from Ni-NTA resin with $15 \mathrm{mM}$ imidazole; 8, fusion protein sample (from elution with $400 \mathrm{mM}$ imidazole) before TEV protease cleavage; 9 , fusion protein sample after TEV protease cleavage showing almost complete cleavage of fusion protein from His6-MBP. (d) Semi-preparative $\mathrm{C}_{4}$ RP-HPLC chromatogram. Absorbance was monitored at 214 and $280 \mathrm{~nm}$. Peak corresponding to correctly folded $\mathrm{rHm} 1 \mathrm{~b}$ is shaded in orange. (e) Analytical $\mathrm{C}_{18}$ RP-HPLC chromatogram resulting from final purification of rHm1b. (f) MALDITOF mass spectrum of $\mathrm{rHm} 1 \mathrm{~b}$. Inset shows the monoisotopic $[\mathrm{M}+1 \mathrm{H}]^{1+}$ ion.

Fig. 3. Effect of rHm1b on human Nav1.1-1.7 stably expressed in HEK293 cells. (a) Representative currents of human Nav1.1-1.7 in the presence of vehicle (grey) or $300 \mathrm{nM} \mathrm{rHm} 1 \mathrm{~b}$ (orange). Inward sodium currents were measured using a holding potential of $-80 \mathrm{mV}$, followed by 
a pre-pulse of $-120 \mathrm{mV}$ for $200 \mathrm{~ms}$ and then a 50-ms step depolarisation to $0 \mathrm{mV}$. In the presence of rHm1b, Nav1.1 and Nav1.3 channels retained sustained currents at the end of a depolarising pulse (indicated by orange shading). (b) Relative current amplitudes of Nav1.1 ( $n=6$; $\mathrm{P}<0.0005$ ), Nav1.3 ( $n=6$; P < 0.005), Nav1.5 ( $n=5 ; \mathrm{P}<0.05)$, and Nav1.7 $(n=5 ; \mathrm{P}<0.05)$ at $5 \mathrm{~ms}$ after the peak current in the presence of vehicle and $300 \mathrm{nM} \mathrm{rHm1b}$. (c) Enlargement of the rHm1b-induced currents of Nav1.5 and Nav1.7 for the same cells shown in (a). Both currents decayed rapidly to zero within $10 \mathrm{~ms}$. (d) Sustained currents at two different time points shown for Nav1.1 and Nav1.3 in the presence of vehicle, $3 \mathrm{nM}, 30 \mathrm{nM}$ and $300 \mathrm{nM} \mathrm{rHm} 1 \mathrm{~b}$. (e) Concentration-response curves for $\mathrm{rHm} 1 \mathrm{~b}$ effect on sustained currents of Nav1.1 $\left(\mathrm{EC}_{50}=11.6 \pm 3.5 \mathrm{nM} ; n=6\right)$ and $\mathrm{Nav} 1.3\left(\mathrm{EC}_{50}=\right.$ $11.8 \pm 1.8 \mathrm{nM} ; n=6$ ). The sustained current (30 ms from peak current) was first normalised to peak current to quantify the magnitude of the effect, then normalised to the data at the highest $\mathrm{rHm} 1 \mathrm{~b}$ concentration. Data were fitted to the Hill equation. (f) Unnormalised concentration-response relationship for sustained currents evoked by rHm1b. The sustained Nav1.1 current $(21.4 \pm 1.4 \%$ of peak current) was 2.5-fold higher than that of Nav1.3 (8.2 $\pm 1.4 \%$ of peak current) after treatment with $300 \mathrm{nM} \mathrm{rHm1b}(\mathrm{P}<0.0005)$. (g) Time course of the Nav1.1 peak currents for the same cell shown in (a) upon application of increasing concentrations of rHm1b. (h) Fitting the Hill equation to concentration-response curves showing peak current inhibition by rHm1b. Peak current amplitude recorded after $\mathrm{rHm} 1 \mathrm{~b}$ treatment was normalised to the maximum peak current from the same cell in the absence of the peptide for Nav1.1-Nav1.7 $(n=3-6)$. At $300 \mathrm{nM}, \mathrm{rHm} 1 \mathrm{~b}$ increased the peak current amplitudes of Nav1.1 (orange) and Nav1.3 (blue) by $32.3 \pm 2.7 \%$ and $34.6 \pm 1.9 \%$, respectively (right panel). Data are mean \pm s.e.m. $* \mathrm{P}<0.05$, **P $<0.005$, ***P $<0.0005$, using paired or unpaired two-tailed Student's $t$-test.

Fig. 4. Effect of nHm1b on human Nav1.1-1.7 stably expressed in HEK293 cells. (a) Representative currents of human Nav1.1-1.7 in the presence of vehicle (grey) or $300 \mathrm{nM} \mathrm{nHm1b}$ (blue). Inward sodium currents were measured using a holding potential of $-80 \mathrm{mV}$, followed by a pre-pulse of $-120 \mathrm{mV}$ for $200 \mathrm{~ms}$ and then a 50-ms step depolarisation to $0 \mathrm{mV}$. (b) Relative current amplitudes of Nav1.1 ( $n=6$; $\mathrm{P}<0.0005)$, Nav1.2 $(n=5 ; \mathrm{P}<0.05)$, Nav1.3 $(n=6 ; \mathrm{P}<$ $0.005), \mathrm{Nav1.5}(n=6 ; \mathrm{P}<0.0005)$ and Nav1.7 $(n=6 ; \mathrm{P}<0.0005)$ at $5 \mathrm{~ms}$ after the peak current following treatment with $\mathrm{nHm} 1 \mathrm{~b}(300 \mathrm{nM})$. (c) Enlargement of the $\mathrm{nHm} 1 \mathrm{~b}$-induced currents of Nav1.2, Nav1.5, and Nav1.7 for the same cells shown in (a). (d) Fits of the Hill equation to concentration-response data showing dose-dependent inhibitory or enhancing effects of $\mathrm{nHm} 1 \mathrm{~b}$ on Nav1.1-1.7. At a saturating concentration of $1 \mu \mathrm{M}, \mathrm{nHm} 1 \mathrm{~b}$ produced a maximum inhibitory effect $(\sim 50 \%)$ at Nav1.2 ( $\left.\mathrm{IC}_{50}=21.6 \pm 0.8 \mathrm{nM} ; n=5\right)$. Peak current amplitude recorded after $\mathrm{nHm} 1 \mathrm{~b}$ treatment was normalised to the maximum peak current from the same cell in the absence of the 
peptide for Nav1.1-1.7 ( $n=5-6)$. (e) Normalised concentration-response curves for nHm1b effect on sustained currents of Nav1.1 $\left(\mathrm{EC}_{50}=12.1 \pm 1.8 \mathrm{nM} ; n=6\right)$ and $\mathrm{Nav} 1.3\left(\mathrm{EC}_{50}=10.7 \pm 2.8 \mathrm{nM}\right.$; $n=6)$. (f) The Nav1.1 sustained current $(21.3 \pm 1.8 \%)$ was two-fold higher than that of Nav1.3 $(9.2 \pm 0.6 \%)$ after treatment with $300 \mathrm{nM} n \mathrm{~nm} 1 \mathrm{~b}(\mathrm{P}<0.005)$. Data are mean \pm s.e.m. $* \mathrm{P}<0.05$, $* * \mathrm{P}<0.005, * * * \mathrm{P}<0.0005$, using paired or unpaired two-tailed Student's $t$-test.

Fig. 5. Effect of rHm1b on the biophysical properties of human Nav1.1. (a) Voltage dependence of normalised peak conductance in the presence of vehicle (grey; $n=6$ ) and $300 \mathrm{nM} \mathrm{rHm} 1 \mathrm{~b}$ (orange; $n=6$ ). The inset shows the voltage protocol. The smooth curves represent the best fits of a Boltzmann function to the averaged data. (b) Voltage dependence of steady-state inactivation of Nav1.1 before and after addition of $300 \mathrm{nM} \mathrm{rHm1b}(n=6$, both). The inset shows the voltage protocol. Normalised peak currents were plotted against prepulse potentials and fitted with a Boltzmann function. (c) $\mathrm{rHm} 1 \mathrm{~b}(300 \mathrm{nM})$ caused a hyperpolarising shift in the voltage dependence

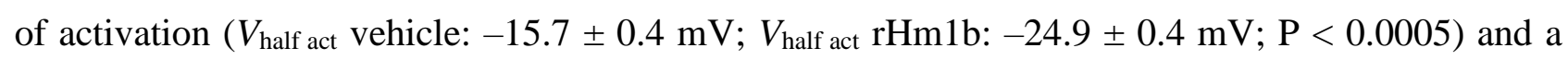
depolarising shift in the voltage dependence of steady-state inactivation ( $V_{\text {half inact }}$ vehicle: $-48.4 \pm$ $\left.0.3 \mathrm{mV} ; V_{\text {half inact }} \mathrm{rHm} 1 \mathrm{~b}:-40.4 \pm 0.3 \mathrm{mV} ; \mathrm{P}<0.05\right)$. (d) The activation slope factor was similar in the presence of $\mathrm{rHm} 1 \mathrm{~b}$ (vehicle: $9.8 \pm 0.5$; $\mathrm{rHm} 1 \mathrm{~b}: 8.4 \pm 0.7 ; \mathrm{P}=0.25$ ), but the inactivation slope factor was significantly increased (vehicle: $4.0 \pm 0.1$; rHm1b: $5.7 \pm 0.4 ; \mathrm{P}<0.05$ ). (e) Overlap of the activation curves (solid line) and steady-state fast inactivation curves (dashed line) showing the window current for Nav1.1 (shaded area) in the presence of vehicle (grey) and rHm1b (orange). (f) Current traces in vehicle condition (grey) and in the presence of $300 \mathrm{nM} \mathrm{rHm} 1 \mathrm{~b}$ (orange). The dashed lines overlaying the vehicle and $\mathrm{rHm} 1 \mathrm{~b}$ traces represent double exponential fit. (g) Effect of $\mathrm{rHm} 1 \mathrm{~b}(300 \mathrm{nM})$ on fast-inactivation time constants for Nav1.1, Nav1.3, Nav1.5 (0.96 $\pm 0.08 \mathrm{~ms}$, $2.09 \pm 0.28 \mathrm{~ms}$ and $0.61 \pm 0.02 \mathrm{~ms}$ respectively; all $\mathrm{P}<0.05)$, and Nav1.7 (0.56 $\pm 0.01 \mathrm{~ms} ; \mathrm{P}=$ 0.06), compared to vehicle. In the presence of $\mathrm{rHm} 1 \mathrm{~b}$, the faster exponential had a relative weight of $0.31 \pm 0.02$ for $\mathrm{Nav}_{1} 1.1,0.31 \pm 0.08$ for $\mathrm{Nav}_{1} 1.3,0.91 \pm 0.02$ for $\mathrm{Nav} 1.5$, and $0.81 \pm 0.07$ for $\mathrm{Na}$ v1.7. (h) Effect of $\mathrm{rHm} 1 \mathrm{~b}(300 \mathrm{nM})$ on slow-inactivation time constants for Nav1.1, Nav1.3, Nav1.5, and Nav1.7 $(6.71 \pm 0.26 \mathrm{~ms}, 7.84 \pm 0.39 \mathrm{~ms}, 4.42 \pm 0.47 \mathrm{~ms}$, and $3.45 \pm 0.22 \mathrm{~ms}$, respectively; all $\mathrm{P}<0.005)$, compared to vehicle. In the presence of $\mathrm{rHm} 1 \mathrm{~b}$, the slower exponential had a relative weight of $0.50 \pm 0.01$ for Nav1.1, $0.64 \pm 0.05$ for Nav1.3, $0.15 \pm 0.02$ for Nav1.5, and $0.25 \pm 0.05$ for Nav1.7. Data are mean \pm s.e.m. $* \mathrm{P}<0.05$, $* * \mathrm{P}<0.005$, ***P $<0.0005$, n.s., not significant, paired two-tailed Student's $t$-test.

Fig. 6. Effect of rHm1b on recovery of human Nav1.1 from fast inactivation. (a) Representative traces illustrating recovery of Nav1.1 from fast inactivation. Upper panel shows the voltage 
protocol used (30 ms to $-120 \mathrm{mV} ; 30 \mathrm{~ms}$ conditioning pulse to $0 \mathrm{mV}$ pulse of variable duration to $-120 \mathrm{mV}$; $30 \mathrm{~ms}$ test pulse to $0 \mathrm{mV}$; step back to $-120 \mathrm{mV}$ ). (b) Ratio of peak amplitude evoked by the test pulse to that evoked by the conditioning pulse, versus duration of inter-pulse interval $(n=10$, both). Curves represent a double-exponential fit, generating fast and slow recovery time constants. (c) There were significant differences in the fast recovery time constants (top panel vehicle: $0.9 \pm 0.1 \mathrm{~ms} ; \mathrm{rHm} 1 \mathrm{~b}$ : $0.5 \pm 0.1 \mathrm{~ms} ; \mathrm{P}<0.0005)$ and slow recovery time constants (bottom panel - vehicle: $10.7 \pm 1.0 \mathrm{~ms} ; \mathrm{rHm} 1 \mathrm{~b}: 6.8 \pm 0.5 \mathrm{~ms} ; \mathrm{P}<0.005)$. Data are mean \pm s.e.m. $* * \mathrm{P}<0.005, * * * \mathrm{P}<0.0005$, paired two-tailed Student's $t$-test.

Fig. 7. Stability of nHm1a and rHm1b in human CSF and serum. Peptides (final concentration $0.5 \mu \mathrm{M}$ ) were incubated at $37^{\circ} \mathrm{C}$ in human CSF or serum. Aliquots were taken for LC-MS analysis at $0,1,2,3,4,8,12,24,48$ and $72 \mathrm{~h}$. (a) Stability of nHm1a [30], rHm1b, ziconotide, and hANP in human CSF. (b) Stability of nHm1a, rHm1b, ziconotide, and hANP in human serum. Curves represent a two-phase exponential fit. Data are mean \pm s.e.m.

Fig. 8. NMR solution structure of rHm1b. (a) Stereoview of the ensemble of $20 \mathrm{rHm} 1 \mathrm{~b}$ structures overlaid over the backbone heavy atoms (PDB 6V6T; BMRB 30695). The N- and C-termini are labelled. The antiparallel $\beta$ strands are coloured orange, whereas the three disulfide bonds are shown in yellow. (b) Schematic of the rHm1b structure determined in this study.

Fig. 9. Surface representation of $\mathbf{r H m} 1 \mathbf{b}$. (a) Primary structures of $\mathrm{nHm} 1 \mathrm{a}$ and $\mathrm{rHm} 1 \mathrm{~b}$. The conserved cysteine residues are shown in bold. Conserved hydrophobic residues are highlighted in red, whereas nonconserved residues are shaded green. Note that the numbering is according to the $\mathrm{rHm} 1 \mathrm{~b}$ sequence. (b) Molecular surface of $\mathrm{rHm} 1 \mathrm{~b}$ with positive and negative charges shown in blue and red, respectively. (c) Molecular surface of rHm1b highlighting the hydrophobic residues (red). The hydrophobicity was calculated according to the Eisenberg hydrophobicity scale [63]. (d) Molecular surface of $\mathrm{rHm} 1 \mathrm{~b}$ highlighting the residues that are different in nHm1a (green). The molecular orientation is the same in panels $(\mathbf{b})$ and $(\mathbf{c})$.

\section{Fig 10. Comparison of primary, secondary, and tertiary structures of nHm1a, rHm1b, and} SGTx1. (a) Primary structures of nHmla, rHm1b, and SGTx1. The locations of the $\beta$ strands are denoted by arrows above the sequences and coloured according to (b) and (c). Note that the numbering is according to the $\mathrm{rHm} 1 \mathrm{~b}$ sequence. (b) Overlay of the $\mathrm{rHm} 1 \mathrm{~b}$ structure determined in this study (PDB 6V6T; orange) with the previously determined structures of nHm1a (PDB 2N6O; cyan) and SGTx1 structures (PDB 1LA4; magenta). The $\mathrm{N}$ - and C-termini are labelled. 
(c) Schematic representations of the $\beta$ hairpins of nHmla (left panel), $\mathrm{rHm} 1 \mathrm{~b}$ (middle panel), and SGTx1 (right panel). Residues making up the $\beta$-strands are coloured and bold, and the hydrogen bonds between $\beta$ strands are shown as dotted lines. The nitrogen, oxygen, and hydrogen atoms are coloured blue, red, and white, respectively.

Table 1 Structural statistics for the ensemble of Hm1b (PDB 6V6T) ${ }^{\mathrm{a}}$

\begin{tabular}{|c|c|}
\hline PDB ID & $6 \mathrm{~V} 6 \mathrm{~T}$ \\
\hline \multicolumn{2}{|l|}{ Experimental restraints } \\
\hline \multicolumn{2}{|l|}{ Inter-proton distance restraints } \\
\hline Total & 499 \\
\hline Intra-residue $(i=j)$ & 114 \\
\hline Sequential $(|i-j|=1)$ & 141 \\
\hline Medium range $(1<|i-j|<5)$ & 96 \\
\hline Long range $(|i-j| \geq 5)$ & 148 \\
\hline Disulfide bond restraints & 9 \\
\hline \multicolumn{2}{|l|}{ Dihedral-angle restraints $(\phi, \psi)$} \\
\hline Total & 22 \\
\hline$\phi$ dihedral angle restraints & 22 \\
\hline$\psi$ dihedral angle restraints & 9 \\
\hline Total number of restraints per residue & 18.2 \\
\hline Violations of experimental restraints & 0 \\
\hline \multicolumn{2}{|l|}{ RMSD from mean coordinate structure $(\AA)^{\mathrm{b}}$} \\
\hline All backbone atoms & $0.28 \pm 0.11$ \\
\hline All heavy atoms & $0.62 \pm 0.13$ \\
\hline Backbone atoms (Residue 3-33) & $0.06 \pm 0.03$ \\
\hline Heavy atoms (Residue 3-33) & $0.38 \pm 0.04$ \\
\hline \multicolumn{2}{|l|}{ Stereochemical quality $^{\mathrm{c}}$} \\
\hline \multicolumn{2}{|l|}{ Ramachandran plot statistics } \\
\hline $\begin{array}{l}\text { Residues in most favored Ramachandran region } \\
(\%)\end{array}$ & $86.5 \pm 2.7$ \\
\hline Disallowed regions $(\%)$ & $0.0 \pm 0.0$ \\
\hline Unfavorable sidechain rotamers $(\%)$ & $2.7 \pm 0.6$ \\
\hline Clashscore, all atoms ${ }^{\mathrm{d}}$ & $0.0 \pm 0.0$ \\
\hline Overall MolProbity score & $1.51 \pm 0.09$ \\
\hline
\end{tabular}

${ }^{\text {a }}$ All statistics are given as mean \pm S.D. 
${ }^{b}$ Mean RMSD calculated over the entire ensemble of 20 structures.

${ }^{\mathrm{c}}$ Sterochemical quality according to MolProbity (http://helix.research.duhs.duke.edu).

${ }^{\mathrm{d}}$ Clashscore is defined the number of steric overlaps $>0.4 \AA$ per 1000 atoms.

Figure 1

a
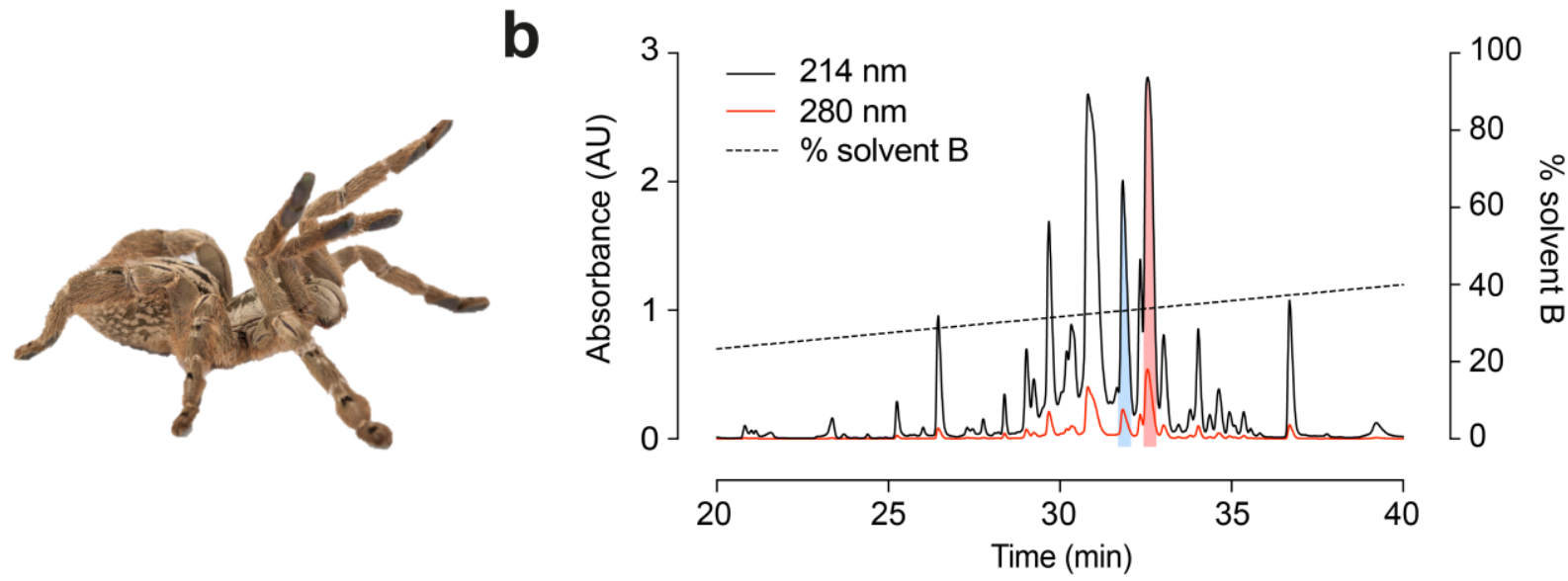

C
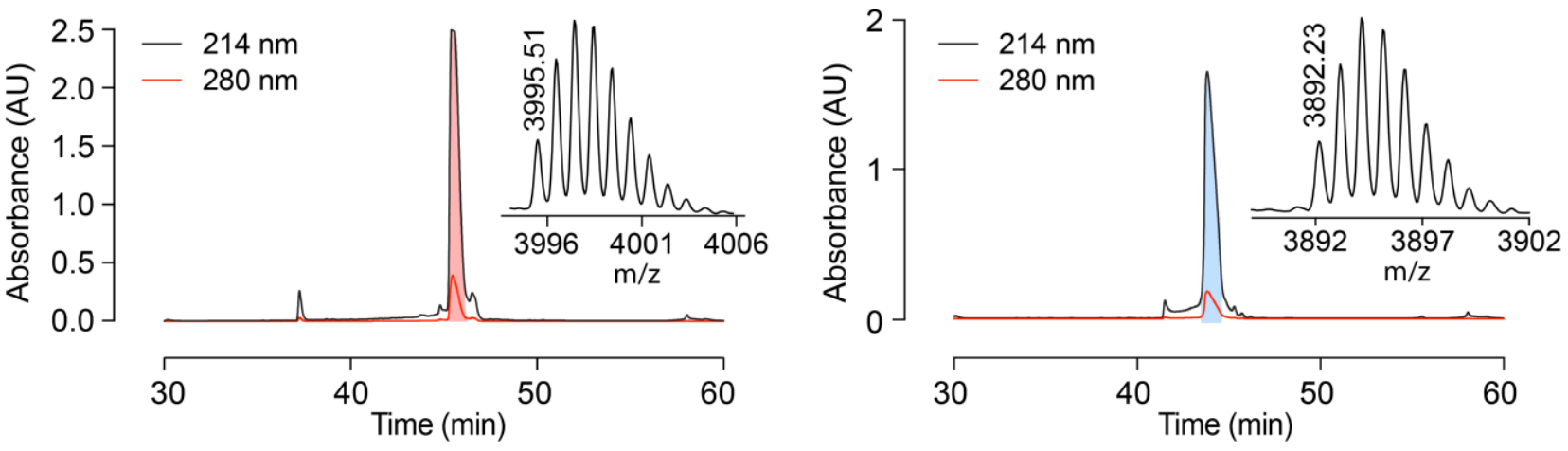

d

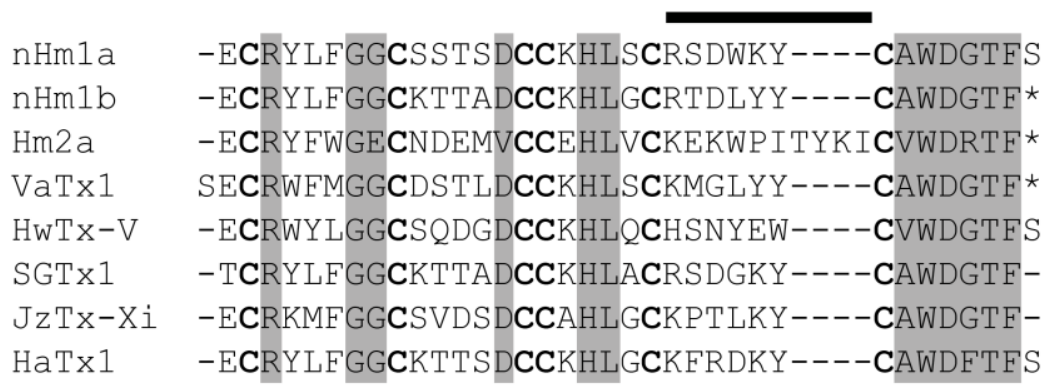

e

\begin{tabular}{lcc}
\multicolumn{1}{c}{ Toxin pair } & $\begin{array}{l}\text { Identity } \\
(\%)\end{array}$ & $\begin{array}{l}\text { Similarity } \\
(\%)\end{array}$ \\
\hline nHm1b/nHm1a & 79 & 88 \\
nHm1b/Hm2a & 42 & 50 \\
nHm1b/VaTx1 & 70 & 79 \\
nHm1b/HwTx-V & 55 & 67 \\
nHm1b/SGTx1 & 87 & 90 \\
nHm1b/JzTx-Xi & 67 & 76 \\
nHm1b/HaTx1 & 79 & 85
\end{tabular}




\section{Figure 2}

a

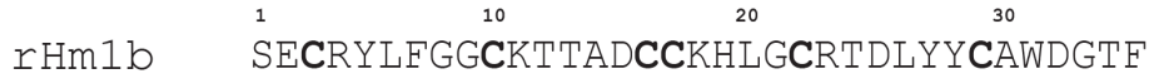

b

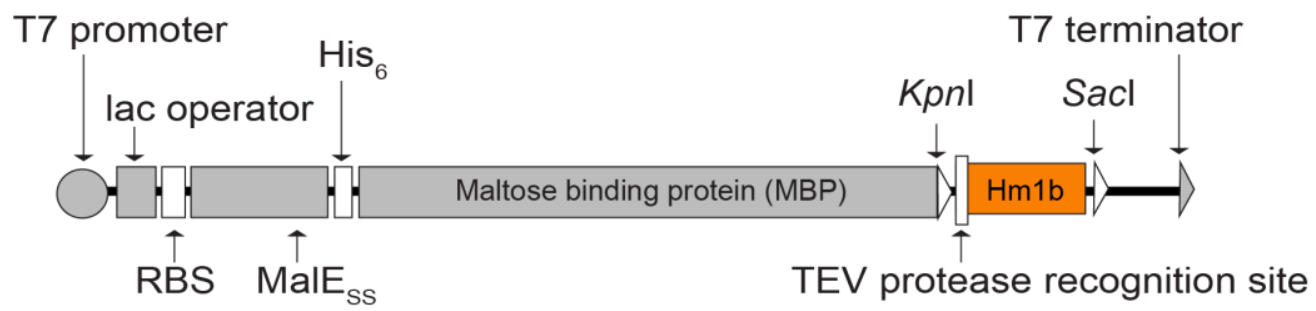

C

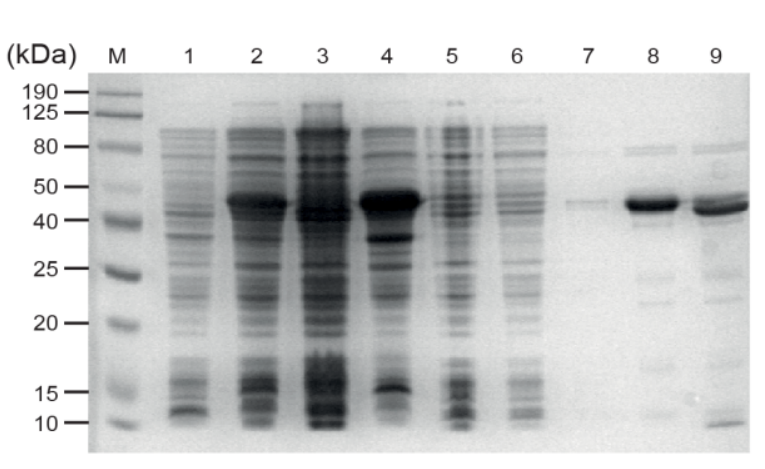

e

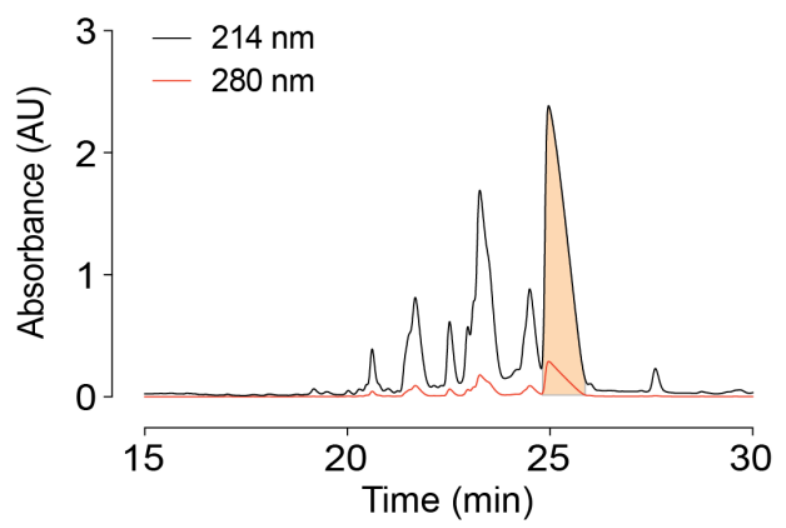

d

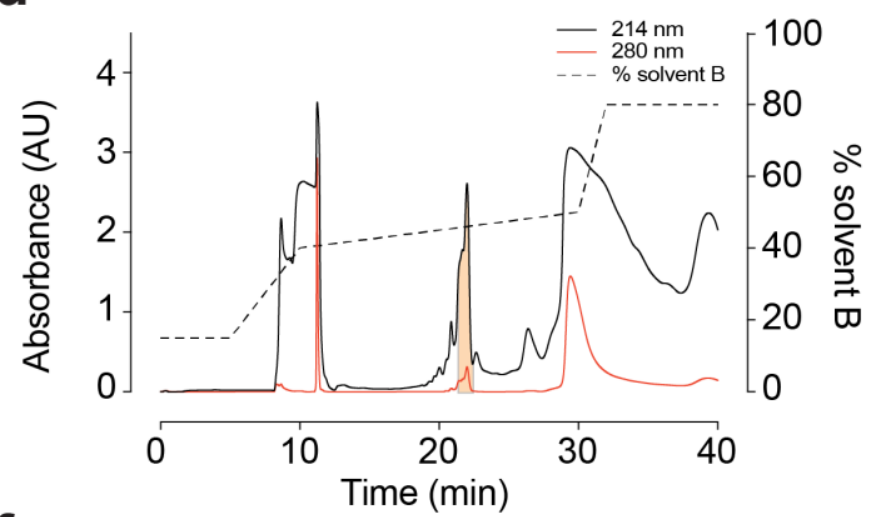

f

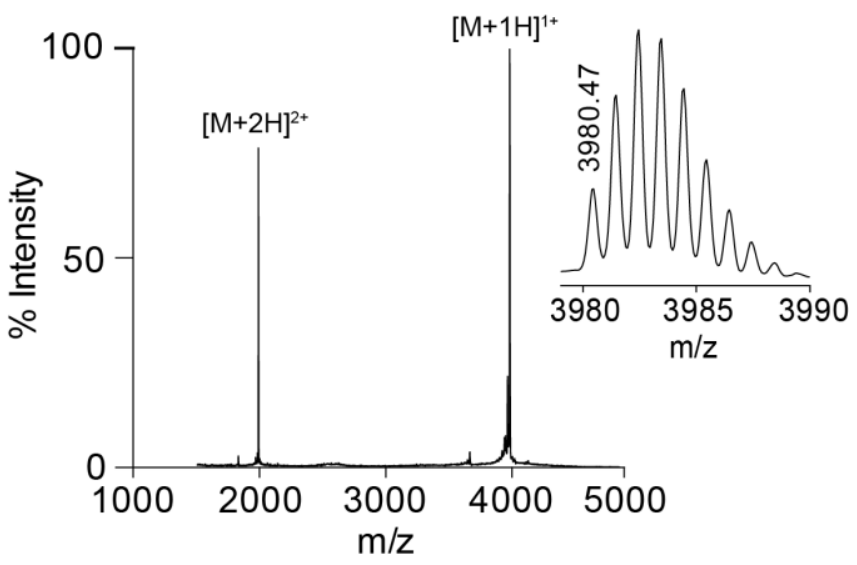




\section{Figure 3}

a
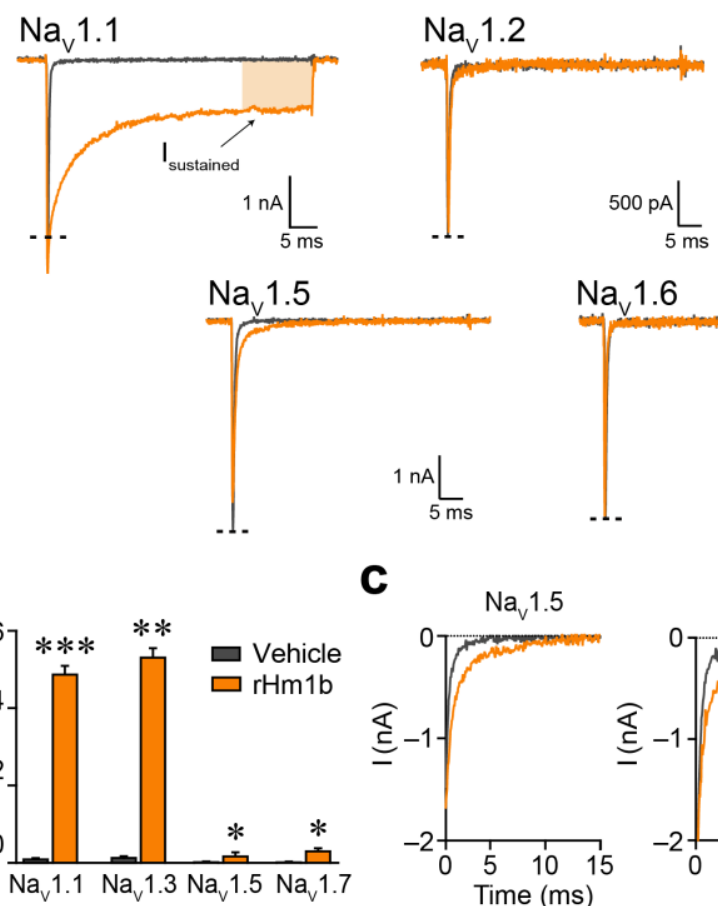

$\mathrm{Na}_{\mathrm{v}} 1.6$
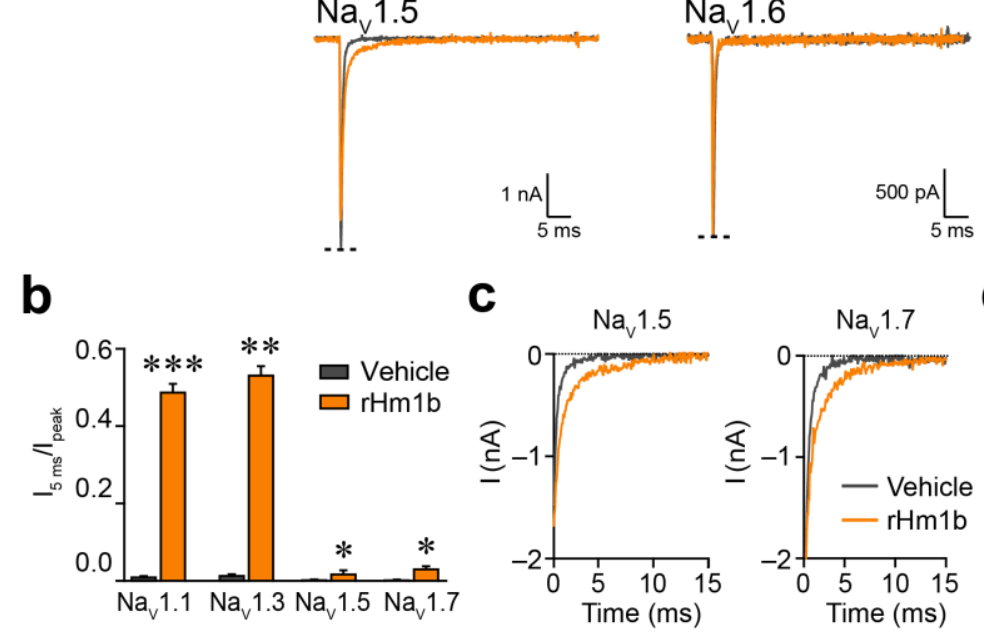
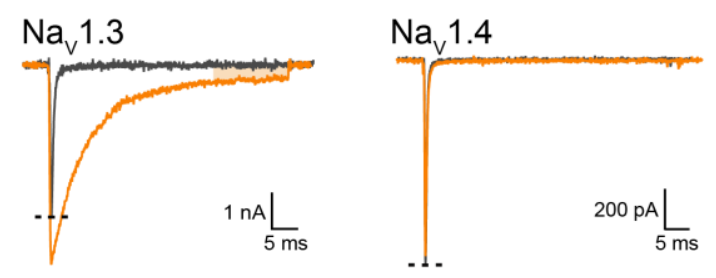

$\mathrm{Na}_{\mathrm{v}} 1.7$

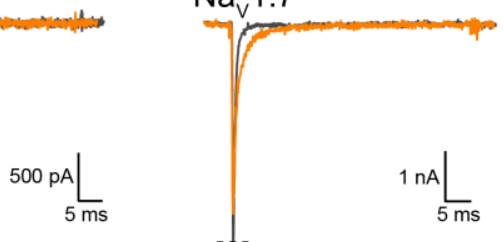

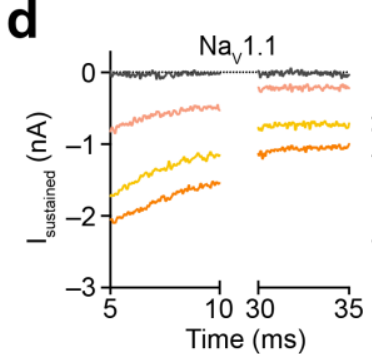

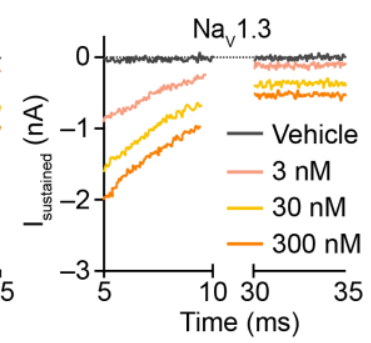

e

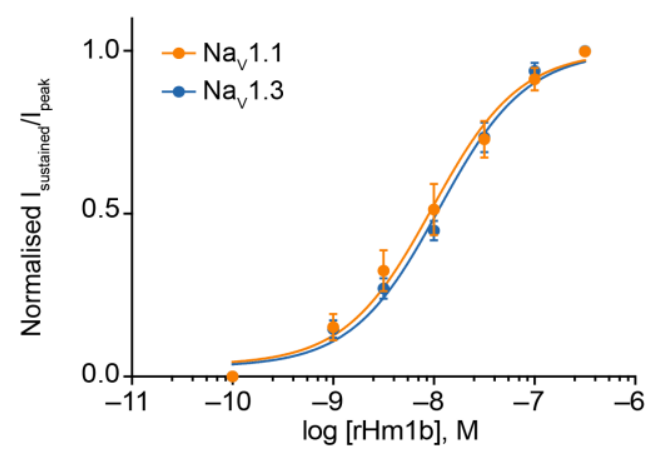

g

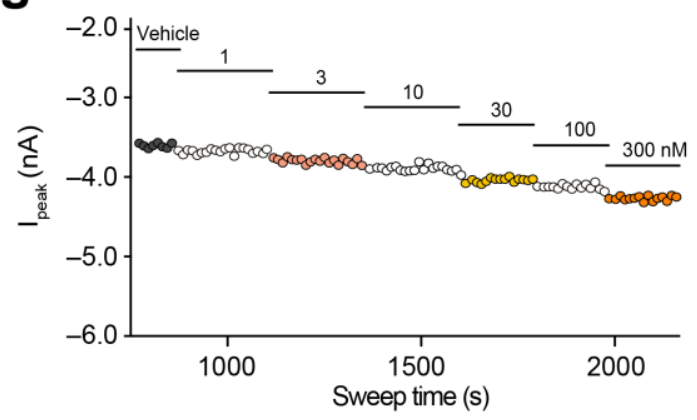

$f$

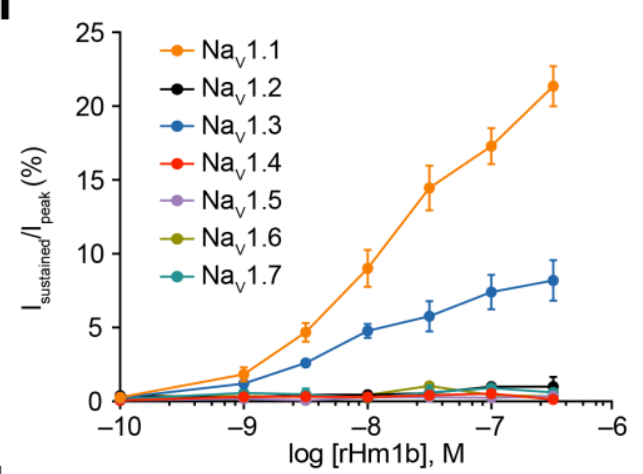

h

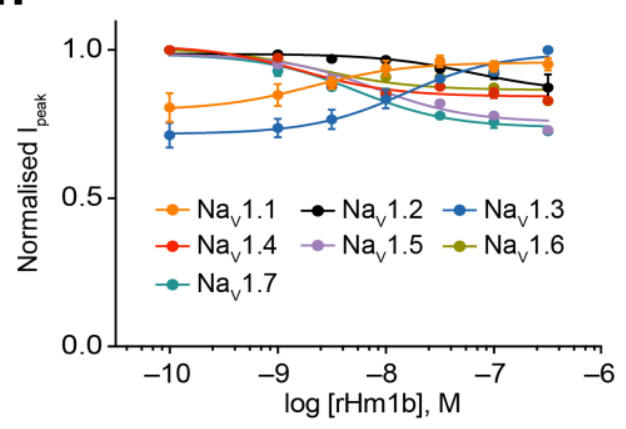

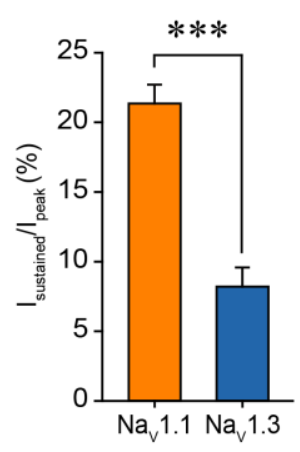

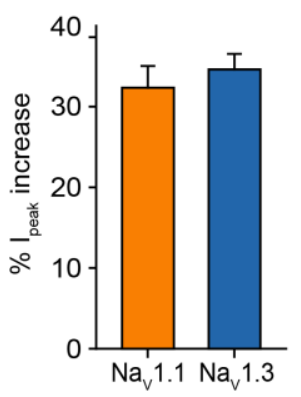




\section{Figure 4}

a
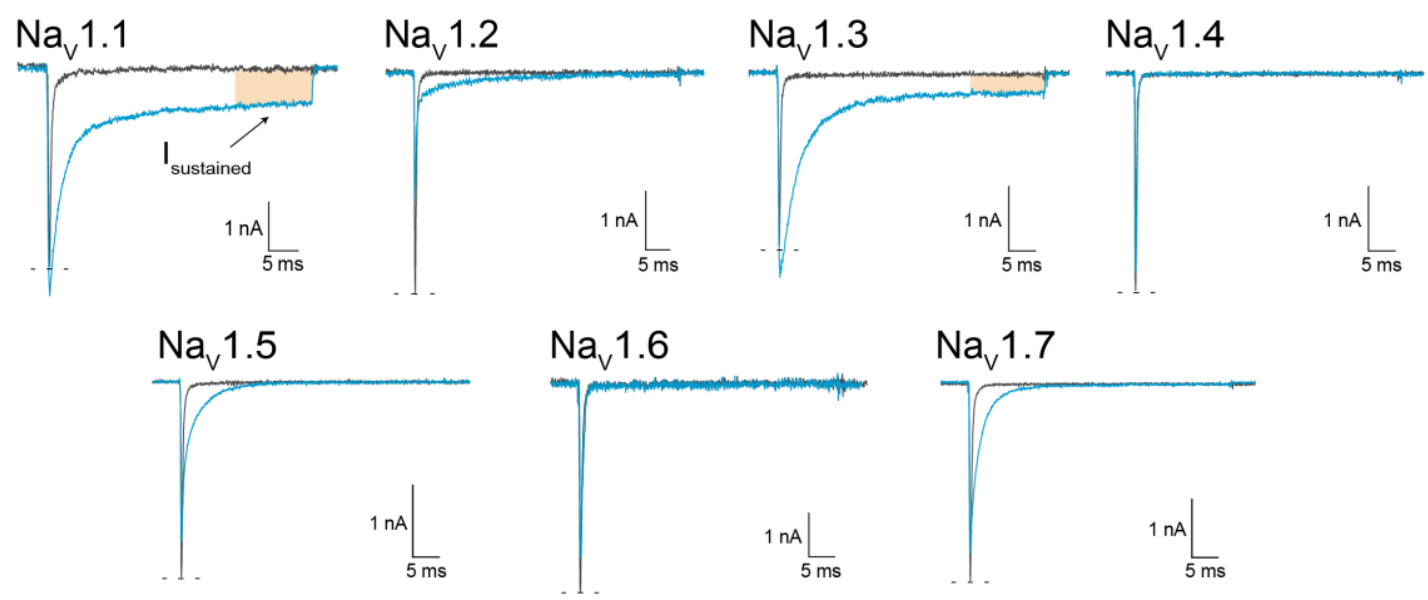

b
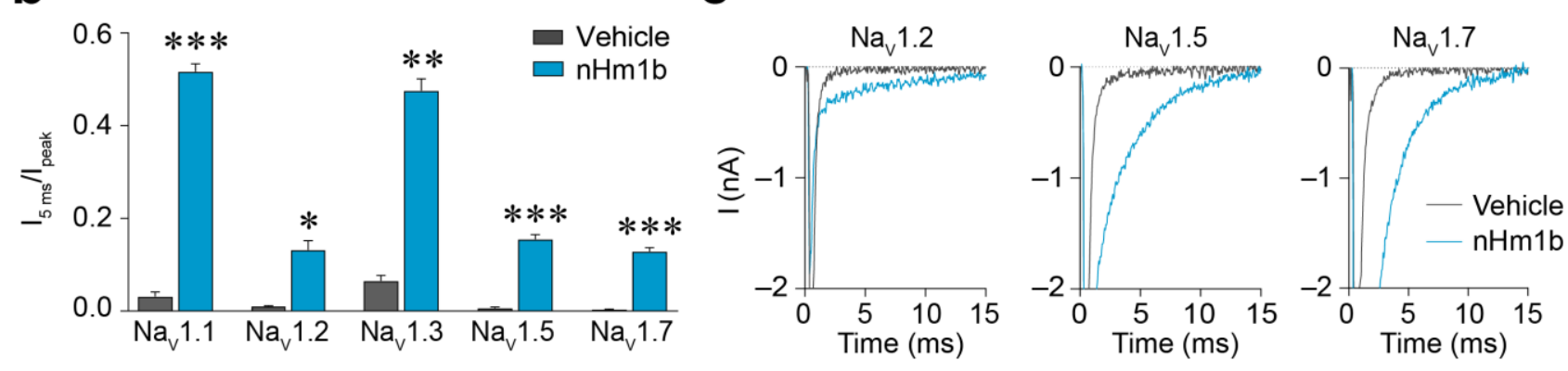

d

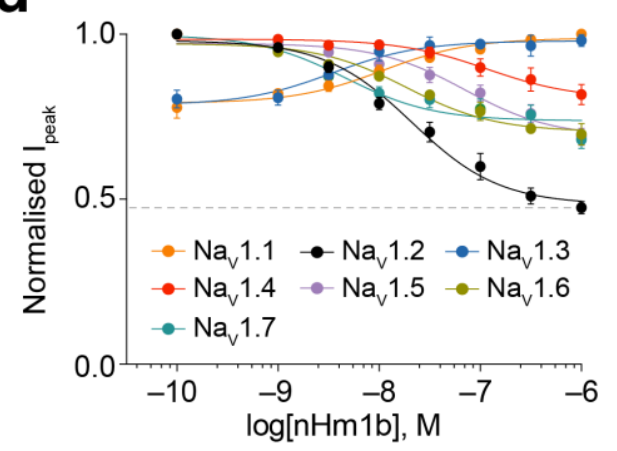

e

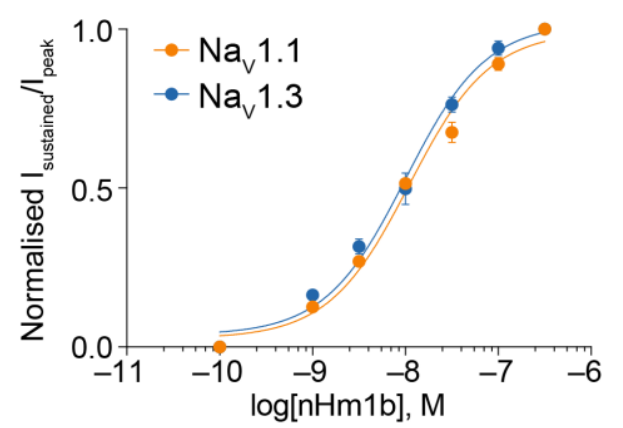

f

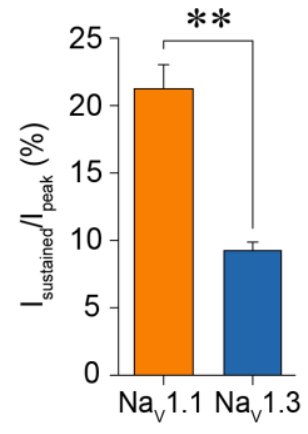




\section{Figure 5}

a

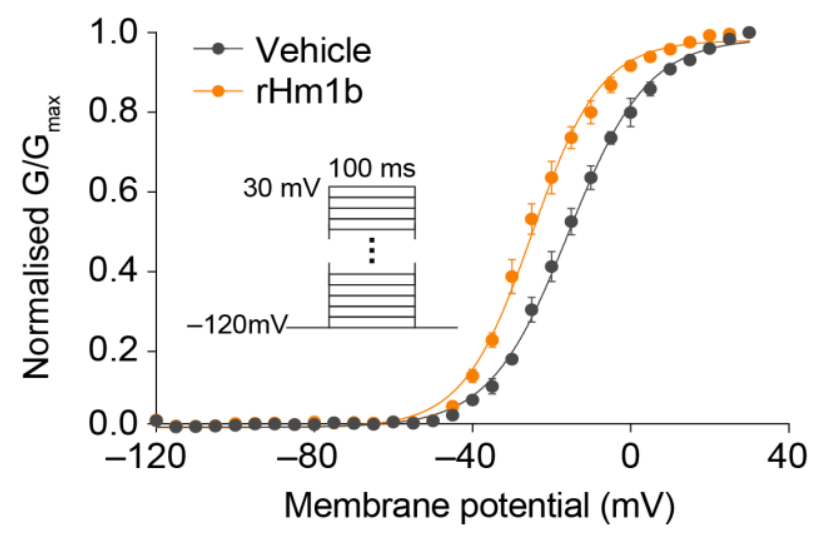

C

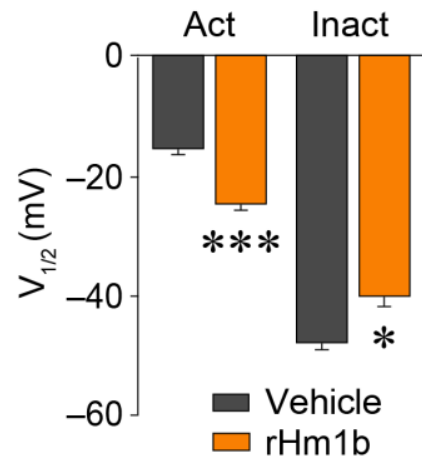

f

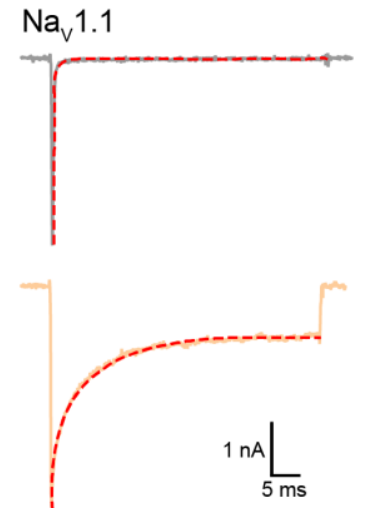

b

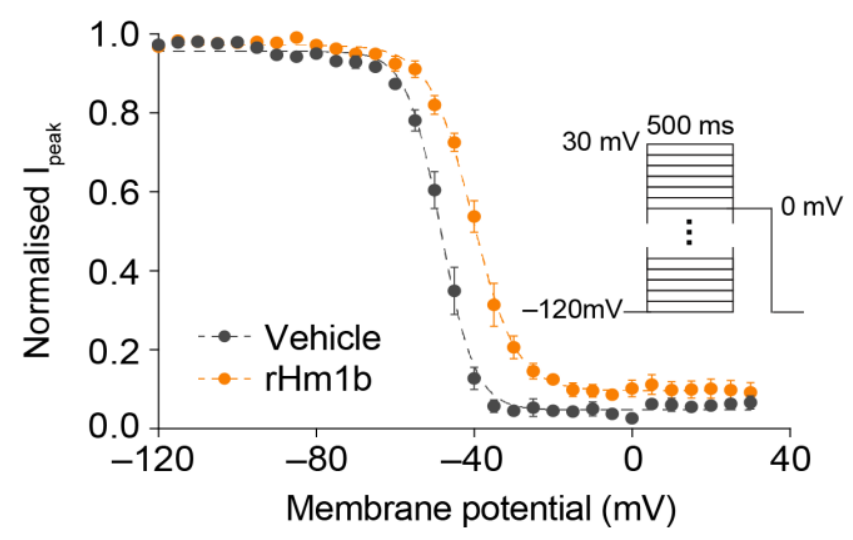

e

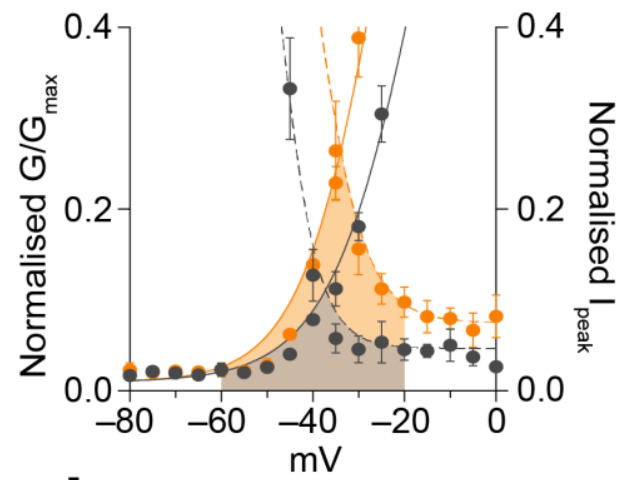

h
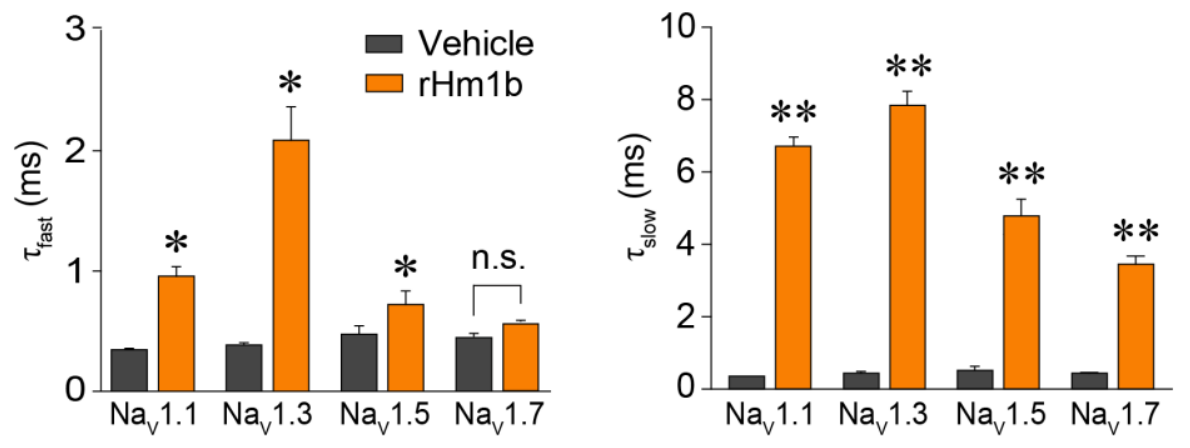


\section{Figure 6}

a

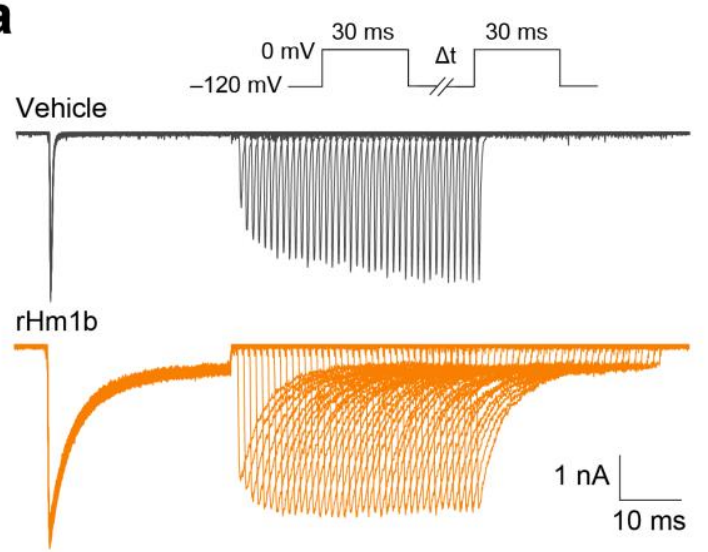

b

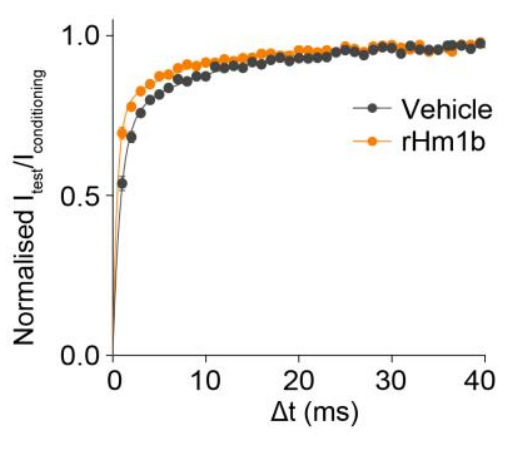

C

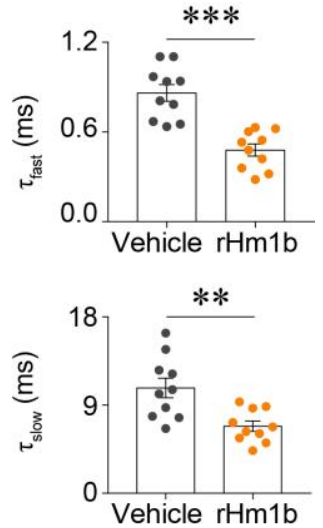




\section{Figure 7}

a

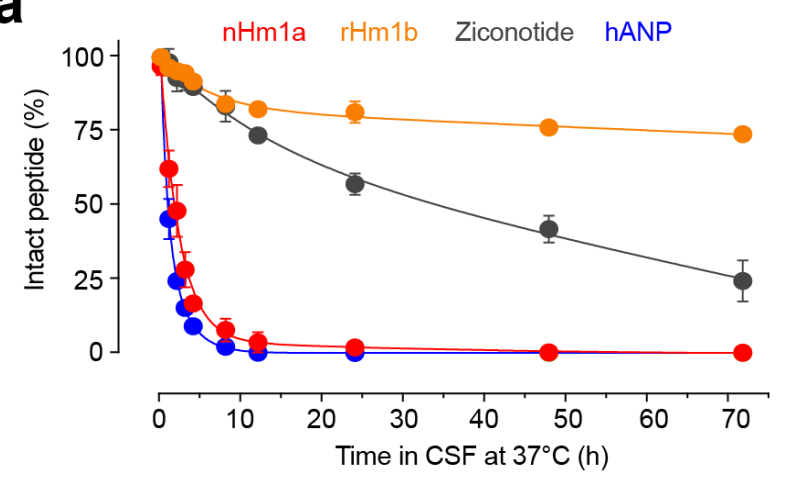

b

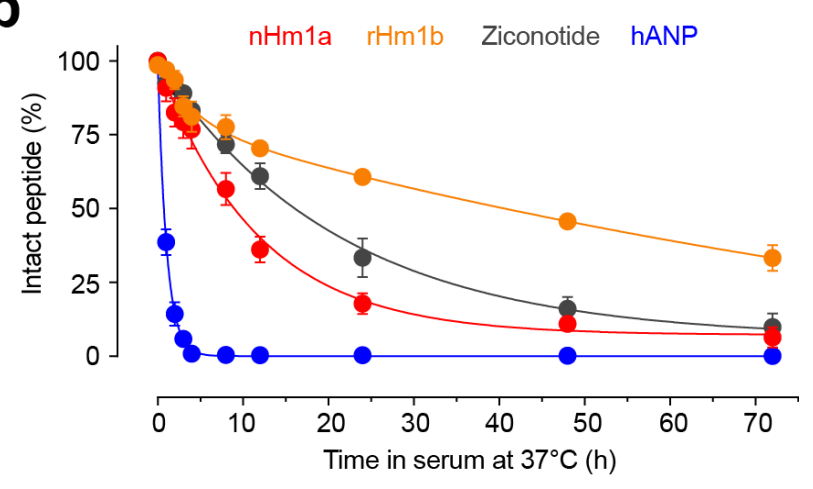


Figure 8

a

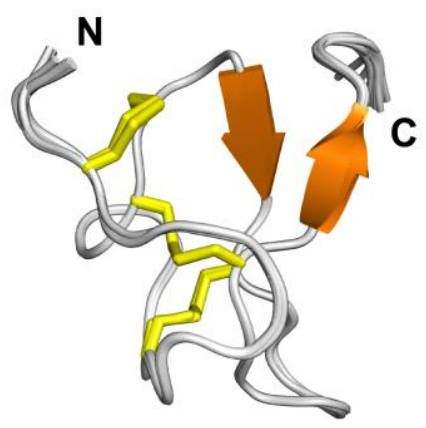

b

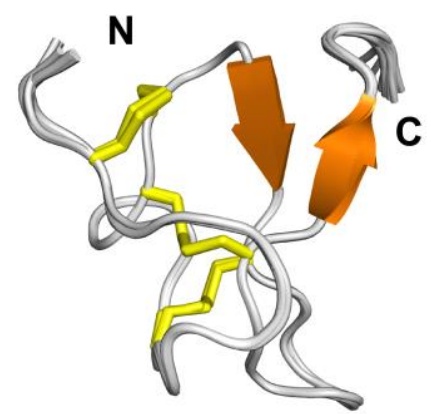

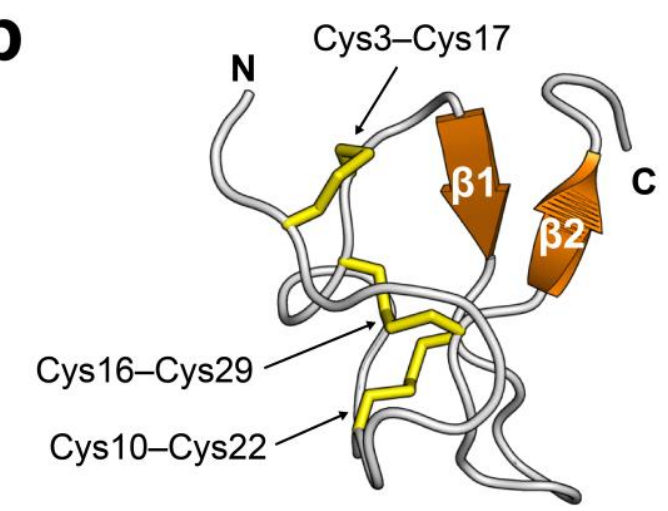


Figure 9

a

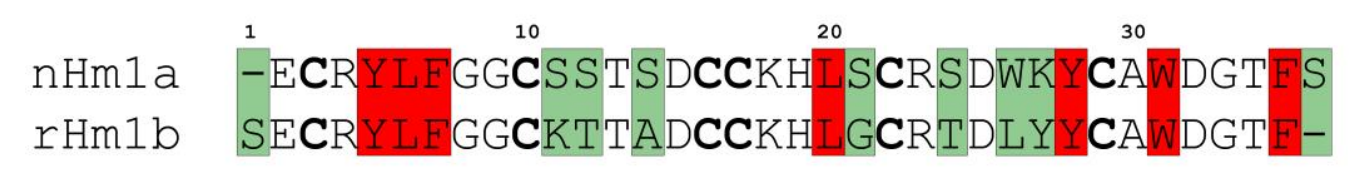

b

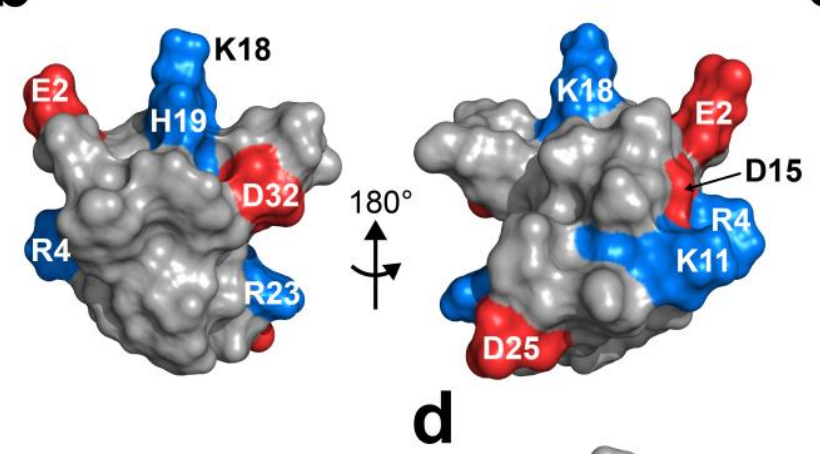

C
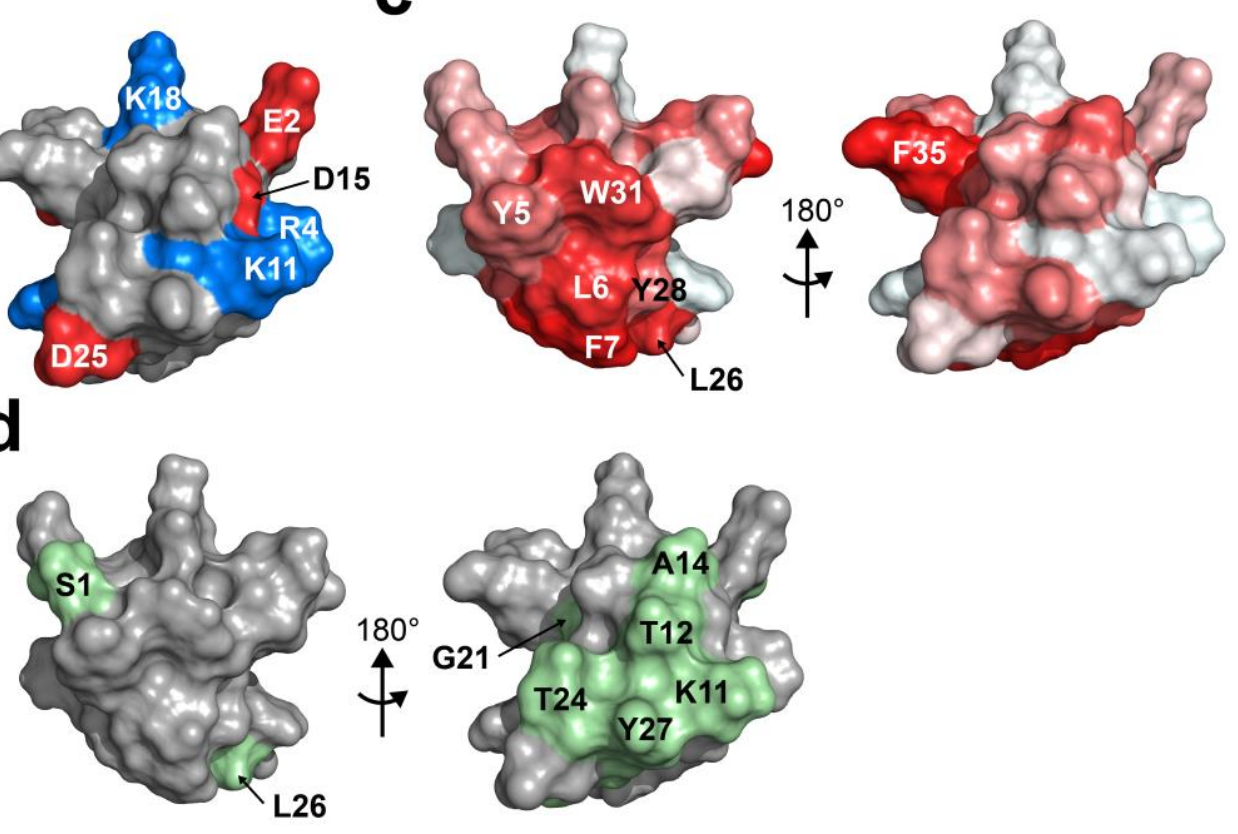
Figure 10

a

nHmla -ECRYLFGGCSSTSDCCKHLSCRSDWKYCAWDGTFS rHm1b SECRYLFGGCKTTADCCKHLGCRTDLYYCAWDGTFSGTX1 -TCRYLFGGCKTTADCCKHLACRSDGKYCAWDGTF10 20 30

b
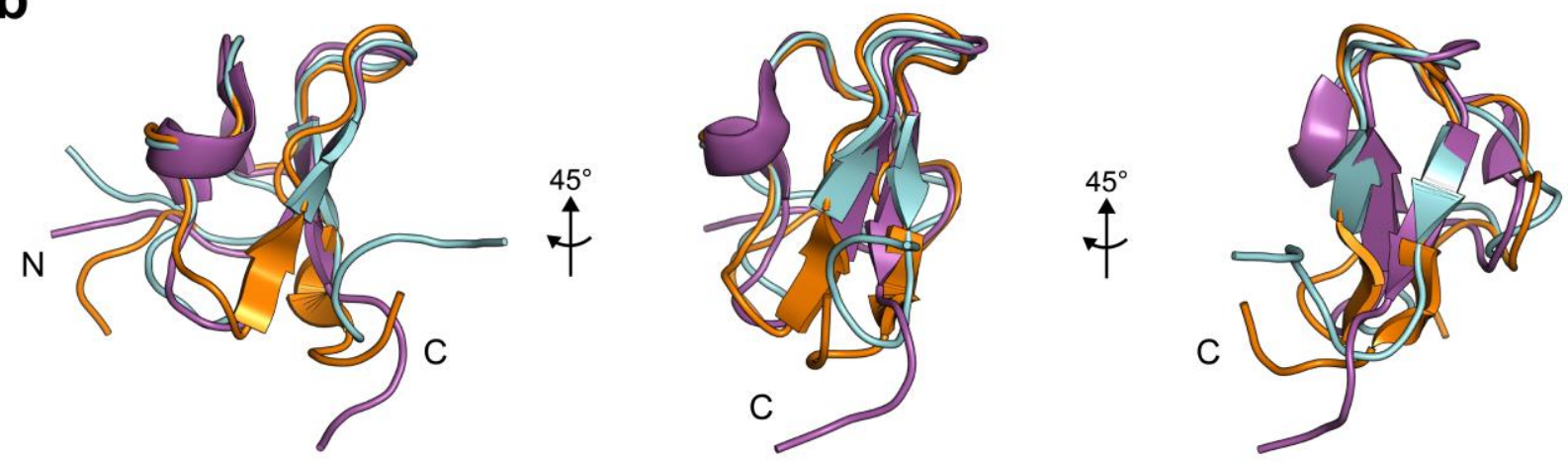

C
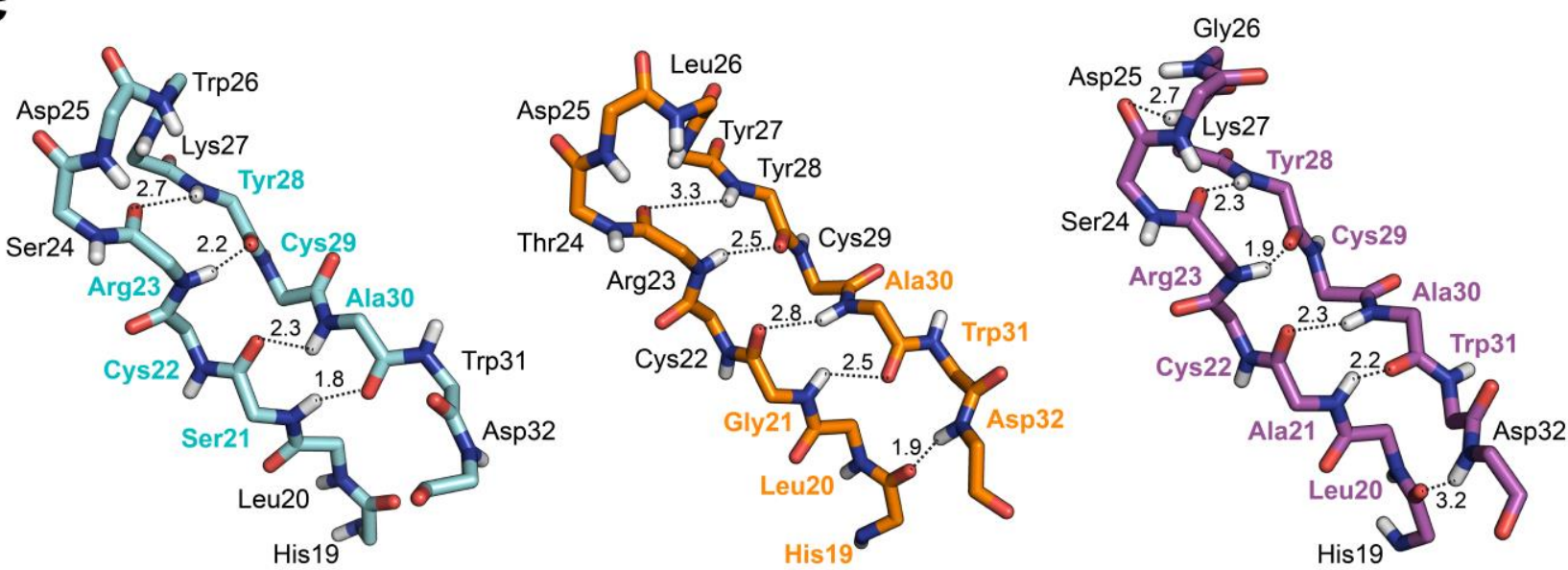